\title{
Aeroelastic Modeling of Elastically Shaped Aircraft Concept via Wing Shaping Control for Drag Reduction
}

\author{
Nhan Nguyen* \\ NASA Ames Research Center, Moffett Field, CA 94035 \\ James Urnes, Sr. $^{\dagger}$ \\ Boeing Research \& Technology, St. Louis, MO 63134
}

\begin{abstract}
Lightweight aircraft design has received a considerable attention in recent years as a means for improving cruise efficiency. Reducing aircraft weight results in lower lift requirements which directly translate into lower drag, hence reduced engine thrust requirements during cruise. The use of lightweight materials such as advanced composite materials has been adopted by airframe manufacturers in current and future aircraft. Modern lightweight materials can provide less structural rigidity while maintaining load-carrying capacity. As structural flexibility increases, aeroelastic interactions with aerodynamic forces and moments become an increasingly important consideration in aircraft design and aerodynamic performance. Furthermore, aeroelastic interactions with flight dynamics can result in issues with vehicle stability and control.

This paper describes a recent aeroelastic modeling effort for an elastically shaped aircraft concept (ESAC). The aircraft model is based on the rigid-body generic transport model (GTM) originally developed at NASA Langley Research Center. The ESAC distinguishes itself from the GTM in that it is equipped with highly flexible wing structures as a weight reduction design feature. More significantly, the wings are outfitted with a novel control effector concept called variable camber continuous trailing edge (VCCTE) flap system for active control of wing aeroelastic deflections to optimize the local angle of attack of wing sections for improved aerodynamic efficiency through cruise drag reduction and lift enhancement during take-off and landing. The VCCTE flap is a multi-functional and aerodynamically efficient device capable of achieving high lift-to-drag ratios. The flap system is comprised of three chordwise segments that form the variable camber feature of the flap and multiple spanwise segments that form a piecewise continuous trailing edge. By configuring the flap camber and trailing edge shape, drag reduction could be achieved. Moreover, some parts of the flap system can be made to have a high frequency response for roll control, gust load alleviation, and aeroservoelastic (ASE) modal suppression control.

The aeroelastic model of the ESAC is based on one-dimensional structural dynamic theory that captures the aeroelastic deformation of a wing structure in a combined motion that involves flapwise bending, chordwise bending, and torsion. The model includes the effect of aircraft propulsion due to wing flexibility which causes the propulsive forces and moments to couple with the wing elastic motion. Engine mass is also accounted in the model. A fuel management model is developed to describe the wing mass change due to fuel usage in the main tank and wing tanks during cruise.

The model computes both static and dynamic responses of the wing structures. The static aeroelastic deflections are used to estimate the effect of wing flexibility on induced drag and the potential drag reduction by the VCCTE flap system. A flutter analysis is conducted to estimate the flutter speed boundary. Gust load alleviation via adaptive control has been recently investigated to address flexibility of aircraft structures. A multi-objective flight control approach is presented for drag reduction control. The approach is based on an optimal control framework using a multi-objective cost function. Future studies will demonstrate the potential benefits of the approach.
\end{abstract}

\section{Introduction}

Air vehicles are typically designed to maintain sufficient structural rigidity for safe load-carrying capacity. Modern engineered materials such as composites have begun to appear in new airframe designs that can provide less structural rigidity while maintaining the same load-carrying capacity. An example of light-weight airframe design is the Boeing

\footnotetext{
*Research Scientist, Associate Fellow AIAA, Intelligent Systems Division, nhan.t.nguyen@nasa.gov, (650)604-4063

${ }^{\dagger}$ Program Manager, Platform Systems/Subsystems Technology, james.m.urnes-sr@ boeing.com, (314)234-3775
} 
787 Dreamliner aircraft, which has highly flexible wing structures than older-generation aircraft. There is a realization that future air vehicle concepts can be developed to take advantage of the structural flexibility afforded by modern engineered materials to improve aerodynamic efficiency.

Elastically shaped aircraft is a concept whereby highly flexible aerodynamic surfaces are elastically shaped in-flight by actively controlling the wing wash-out twist and wing bending deflection in order to change the local angle of attack in such a manner that can result in lower fuel burn by drag reduction during cruise. Moreover, structural flexibility will be leveraged to realize a revolutionary, optimal wing shape design that can accommodate a significant curvature for drag reduction benefits as opposed to a conventional straight, planar wing design. Elastically shaped aircraft, therefore, may be viewed as a biologically-inspired concept that could potentially revolutionize the conventional airframe design. Taking a cue from birds' efficient shape-changing wings, this concept may be able to bring future air vehicle concepts to the next level in terms of performance, efficiency, and maneuverability.

A conceptual study has recently been conducted with the funding support of NASA Innovation Fund Award sponsored by NASA Innovative Partnerships Program in 2010. ${ }^{1}$ The plan of this study is to conduct a multi-disciplinary design, analysis and optimization to examine the potential benefits of the ESAC over a conventional aircraft design. The four major technical areas of the study are:

1. Vehicle conceptual design and optimization - A futuristic elastically shaped wing superimposed on an available commercial transport fuselage is designed by aerodynamic optimization. The optimization will be conducted to identify an optimal non-planar curved wing shape which is defined by the wing curvature and wash-out twist of the elastic wing that minimize induced drag by tailoring the wing lift distribution. A comparative study of the fuel savings will be made using available performance data for a representative commercial transport and engines.

2. Aeroelastic flight dynamic modeling - A coupled aeroelastic flight dynamic model of the ESAC is developed using the GTM for aerodynamics, and stability and control analysis.

3. Elastically wing shaping actuation design - A low drag distributed actuation concept is developed to size and strategically place new aerodynamic surfaces throughout the elastic wing. The distributed control surfaces will be used to actively shape the elastic wing to gain aerodynamic efficiency. Commercially available sensor technology for shape measurement will be leveraged and assumed to be available for wing shaping control.

4. Flight control design and vehicle simulation - A multi-objective flight control system will be developed to simultaneously gain aerodynamic efficiency and maintain traditional pilot command-tracking tasks for guidance and navigation. A guidance law to achieve a low drag objective will be developed for a cruise phase to specify both the desired drag and vehicle attitude objectives for flight control performance. Multi-objective optimal control will be introduced for flight control design. A complete vehicle control simulation will be performed to demonstrate feasibility.

This paper focuses on the areas 2 and 3 which address aeroelasticity and wing shaping control design for the ESAC.

\section{Elastically Shaped Aircraft Concept}

The elastically shaped aircraft concept is modeled as a notional single-aisle, mid-size, 200-passenger aircraft. The geometry of the ESAC is obtained by scaling up the geometry of NASA generic transport model (GTM) by a scale of 200:11. The GTM is a research platform that includes a wind tunnel model and a remotely piloted vehicle as shown in Figure 1. ${ }^{2}$ Figure 2 is an illustration of the GTM planform. The reason for selecting the GTM is that there already exists an extensive wind tunnel aerodynamic database that could be used for validation in the study. The benchmark configuration represents one of the most common types of transport aircraft in the commercial aviation sector that provides short-to-medium range passenger carrying capacities.

The aircraft has a take-off weight of 200,000 lbs for a typical operating load (gear up, flap up) that includes cargo, fuel, and passengers. Fuel weighs about 50,000 lbs for a range of about 3,000 nautical miles.

To compute the mass and inertia properties of the benchmark aircraft, a component-based approach is used. The aircraft is divided into the following components: fuselage, wings, horizontal tails, vertical tail, engines, operational empty weight (OEW) equipment, and typical load including passengers, cargo, and fuel. The fuselage, wings, horizontal tails, and vertical tail are modeled as shell structures with constant wall thicknesses. ${ }^{3}$ Based on publicly available data of component weight breakdown for various aircraft, ${ }^{4}$ an average wing mass relative to the total empty weight of the aircraft is taken to be $24.2 \%$ of the OEW. 
The aircraft is powered by two 44,000-lb rated turbofan engines. The maximum thrust per engine at sea level takeoff is 44,000 lbs and varies linearly to $30,000 \mathrm{lbs}$ at sea level Mach 0.8 The thrust specific fuel consumption (TSFC) at sea level take-off is $0.3460 / \mathrm{hr}$ and varies linearly to $0.6656 / \mathrm{hr}$ at sea level Mach 0.8 . The total maximum engine thrust and TSFC as functions of altitude and Mach number are given by the following formulas:

$$
\begin{gathered}
T_{\max }(h, M)=(-77031 M+100986)\left(1+\frac{\gamma-1}{2} M^{2}\right)^{\frac{\gamma}{\gamma-1}} \delta(h) \\
c(M, h)=(0.45642 M+0.26156) \sqrt{\theta(h)\left(1+\frac{\gamma-1}{2} M^{2}\right)}
\end{gathered}
$$

where $T_{\max }$ is the maximum thrust, $c$ is the TSFC, $M$ is Mach number, $\delta=p / p_{S L}$ is the pressure ratio, $\theta=T / T_{S L}$ is the temperature ratio, and $p_{S L}$ and $T_{S L}$ are the pressure and temperature at sea level.

The TSFC represents the fuel burn rate, hence an important parameter in the estimation of range and fuel burn.

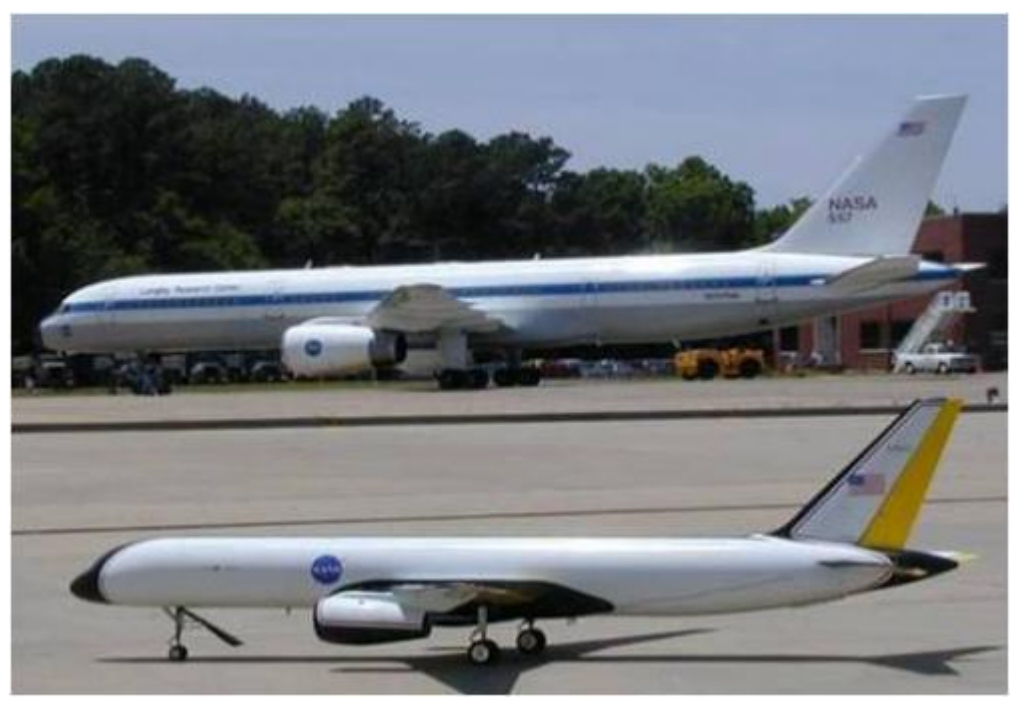

Figure 1 - Generic Transport Model (GTM) and Remotely Piloted Vehicle at NASA Langley

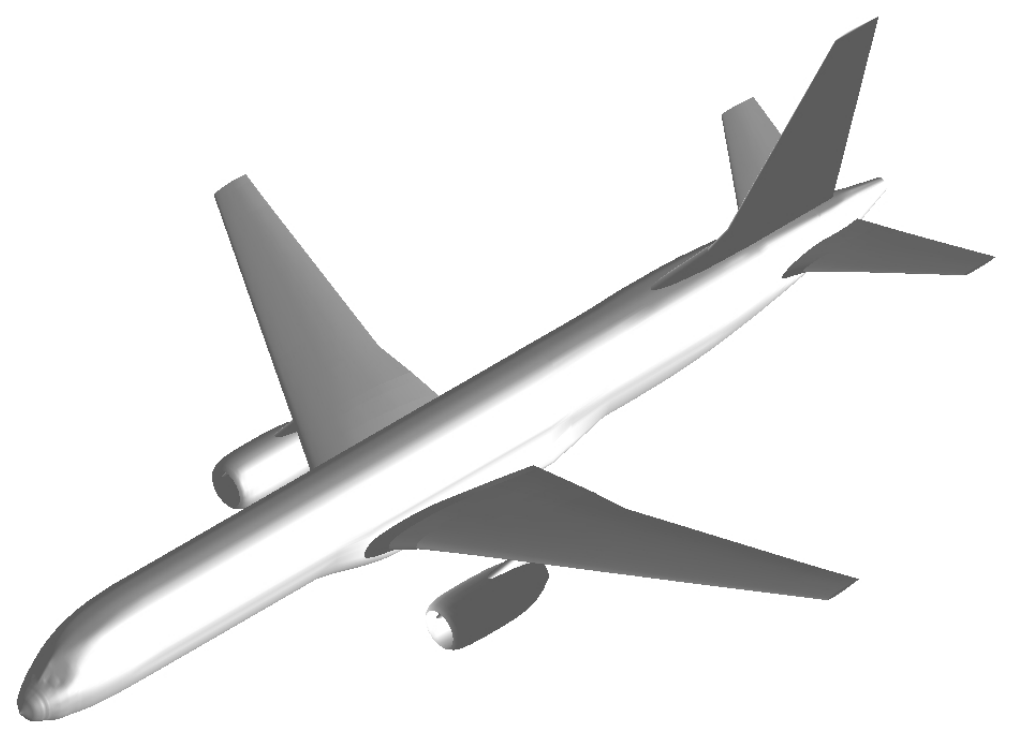

Figure 2 - GTM Planform

The ESAC can be equipped with either conventional planar straight wings or more aerodynamically efficient non-planar curved wings. The conceptual study funded by the NASA Innovative Partnerships Program has explored 
various options of wing shapes through aerodynamic optimization and has produced some interesting aerodynamically efficient concepts. One such wing configuration is an inflected wing shape which could be a candidate for the ESAC. ${ }^{1,5}$
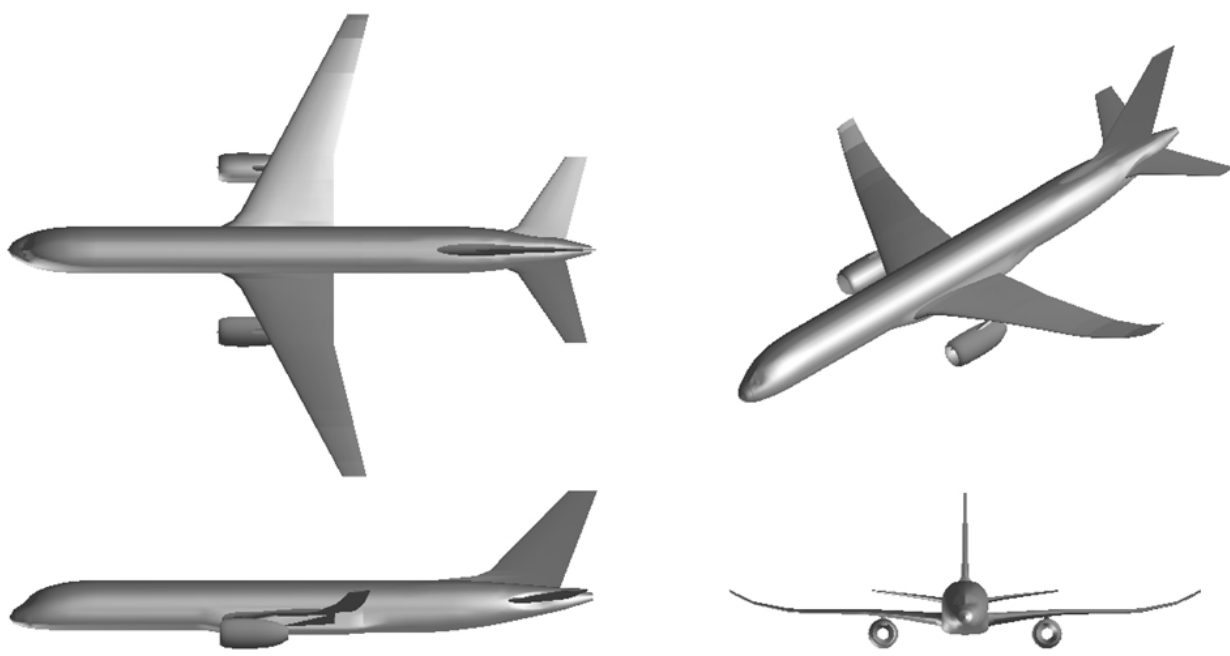

Figure 4 - Inflected-Wing Elastically Shaped Aircraft Concept

\section{Variable Camber Continuous Trailing Flap Concept for Active Wing Shaping Control}

Aeroelastic deflection can affect aircraft aerodynamics. As an aircraft cruises, fuel is burned and the wing loading is reduced, thereby causing the wing shape to change. The change in wing shape can cause a drag penalty since the wing shape no longer retains its optimal design shape. This particularly can be an important issue for lightweight airframes. Thus, aircraft with flexible wing structures can potentially become less fuel-efficient if there is no mechanism to compensate for aeroelastic deflection. Figure 5 illustrates the drag polars estimated by an aerodynamic vortex-lattice model at three points in the cruise envelope corresponding to $80 \%$ fuel, $50 \%$ fuel, and $20 \%$ fuel remaining. ${ }^{1}$ The midpoint of cruise that corresponds to $50 \%$ fuel is considered to be the wing optimal design point. The initial analysis indicate a significant drag penalty as the wing shape moves away from its optimal shape.

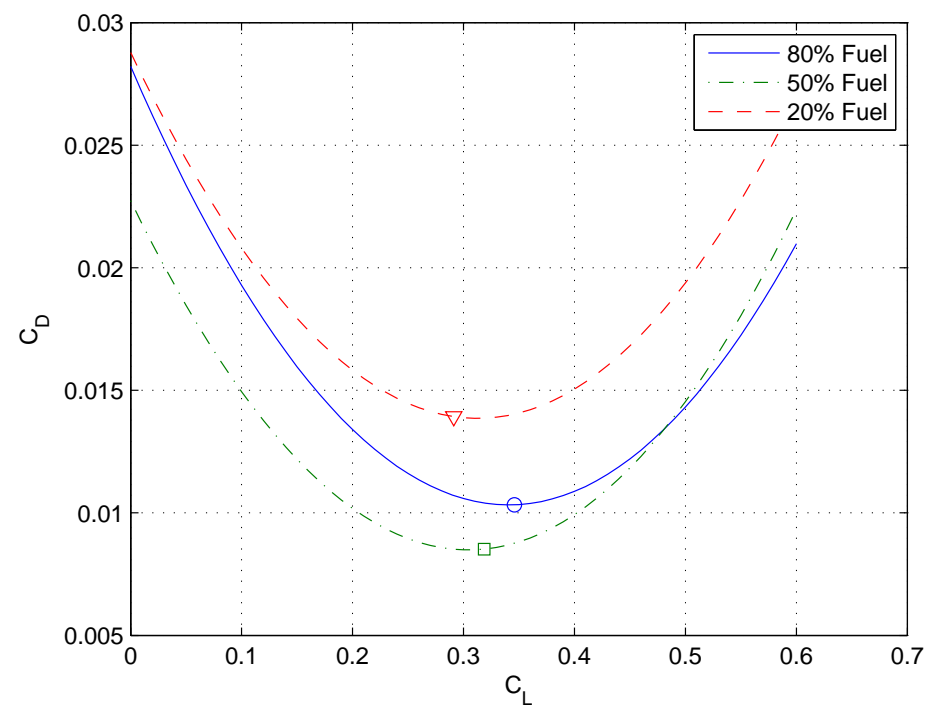

Figure $5-C_{D}$ Penalty due to Aeroelastic Deflection

The objective of wing shaping control is to restore the wing shape back to an optimal shape through flap and or slat deployments. However, the drag penalty due to flap and slat deflections can negate any benefit attained from wing 
shaping control. The conceptual study explored the use of distributed conventional flap and slat systems for wing shaping control. In a conventional flap design, individual flaps are actuated independently. As a result, the trailing edge of a wing formed by the flap deflections is discontinuous. This discontinuity is a source of drag penalty as well as acoustic emissions. Aerodynamic vortex-lattice calculations show that the drag penalty due to the conventional flap system could be substantial. ${ }^{1}$ One way to reduce the drag penalty is to use a single flap surface over a wide wing span. However, this would compromise the flexibility and effectiveness of wing shaping control. Thus, a new, low-drag wing shaping control device has been proposed in the conceptual study and is currently being further developed. This flap system is referred to as a variable camber continuous trailing edge (VCCTE) flap system, as shown in Figures 6 to 8 , which appears to provide a significant drag reduction over a conventional flap system. ${ }^{1}$

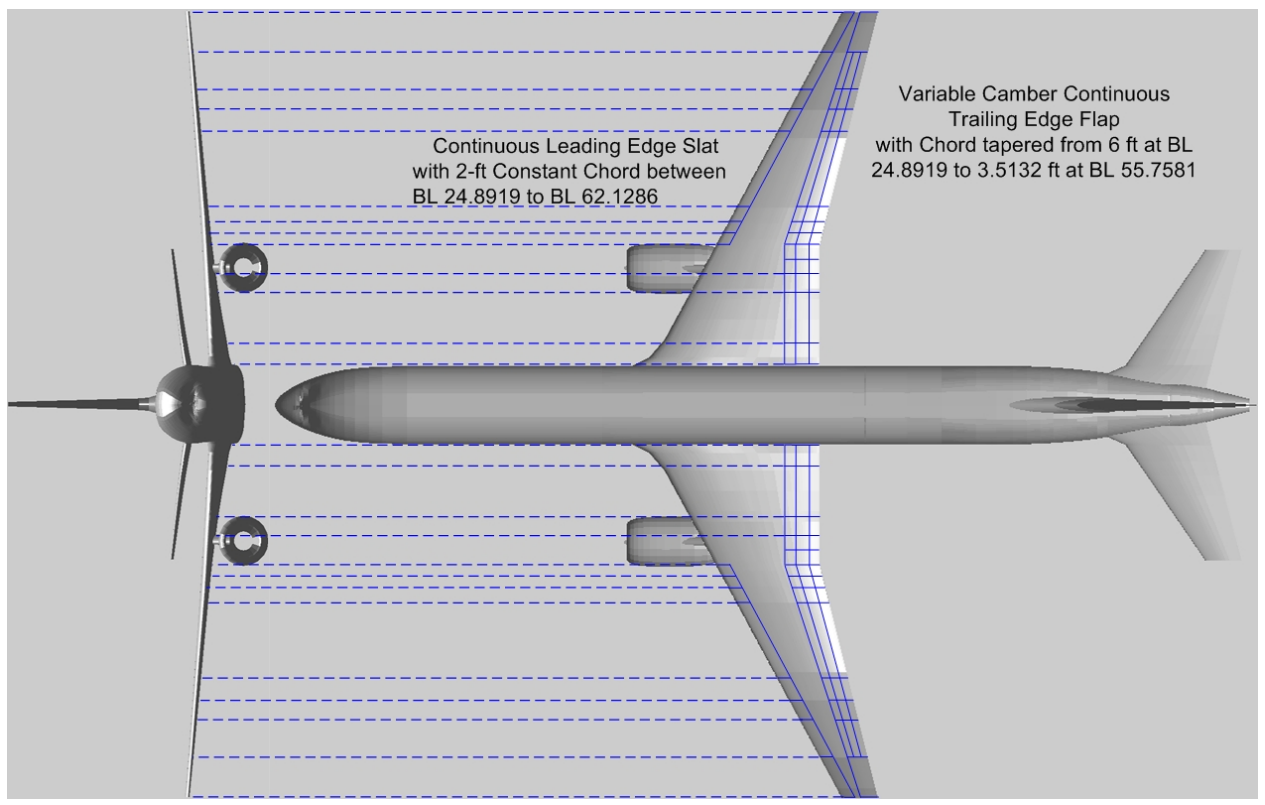

Figure 6 - Planform of Variable Camber Continuous Trailing Edge Flap Concept

The two main features of this flap concept ${ }^{1}$ that can achieve a significant drag reduction are:

1. Variable camber - The flap chord is comprised of three chordwise segments of equal chord length as shown in Figure 7. These three flap segments are actuated in unison when a flap deflection command is given. Each flap segment is deflected by an angle equal to one third of the commanded flap deflection relative to each other. For example, for a commanded flap deflection of $12^{\circ}$, flap segment 1 which is positioned next to the wing is deflected $4^{\circ}$, flap segment 2 that follows flap segment 1 is deflected $8^{\circ}$, and flap segment 3 at the trailing edge is deflected by $12^{\circ}$. Thus

$$
\begin{gathered}
f_{1}=\frac{f_{c}}{3} \\
f_{2}=\frac{2 f_{c}}{3} \\
f_{3}=f_{c}
\end{gathered}
$$

where $f_{c}$ is the commanded flap deflection.

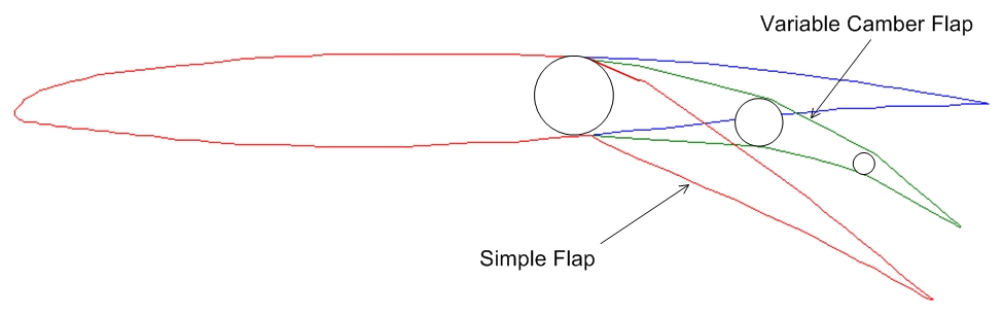


The camber angle of the flap is the difference between between $f_{3}$ and $f_{1}$. Thus, the variable camber angle $\chi=2 f_{c} / 3$ is a function of the commanded flap deflection. A cambered flap is more effective in producing lift than a straight uncambered flap.

The variable camber flap produces about the same downwash as a simple plain flap deflected by the same angle, as seen in Figure 8. However, the normal surface area of the variable camber flap exposed to the flow field is significantly reduced. Thus, the drag reduction benefit of the variable camber flap is realized since the pressure drag across the flap surface is reduced due to less exposed normal surface area.

2. Continuous trailing edge - The continuous trailing edge is comprised of 12 spanwise segments to form a continuous trailing edge when the flap is deflected. This continuous trailing edge would eliminate vortices which otherwise would have formed at the conventional flap discontinuity in the trailing edge region. By reducing or eliminating vortex formation, drag losses as well as acoustic emissions from turbulence could be attenuated. The material and design methodology used to fabricate this flap system must be able to provide some degree of structural compliance due to differential flap deflections that each flap spanwise segment would experience to maintain a continuity of the trailing edge. This feature provides a further drag reduction benefit in addition to the variable camber chordwise flap segmentation concept.

To approximate this continuous trailing edge curve, the continuous trailing edge flap system is assumed to be made up of 12 continuous flap segments where they join together to form a continuous trailing edge. Each flap segment is designed to be actuated in relation to the adjacent flap segments in the spanwise direction to form a continuous trailing edge, as well as in the chordwise direction to form a desired camber. The flap spans the wing trailing edge from BBL $6.2807 \mathrm{ft}$, which is abutted to the fuselage, to BBL $55.7581 \mathrm{ft}$. The flap deflection angle varies continuously from zero at these two BBL stations to the maximum commanded flap deflection at BBL $24.8919 \mathrm{ft}$. The chord is tapered from $6 \mathrm{ft}$ at BBL $24.8919 \mathrm{ft}$ to $3.5132 \mathrm{ft}$ at BBL $55.7581 \mathrm{ft}$, and then remains constant at $6 \mathrm{ft}$ between BBL $6.2807 \mathrm{ft}$ to BBL $24.8919 \mathrm{ft}$.

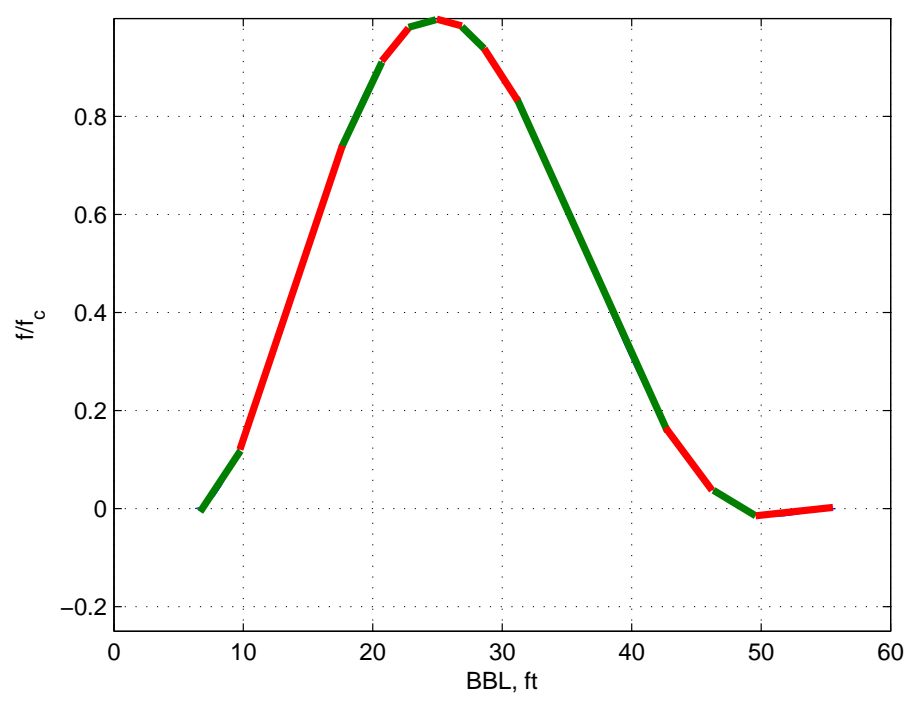

Figure 8 - Continuous Trailing Edge Flap Segments

A theoretical smooth trailing edge shape is generated by a 5th-degree polynomial with zero-displacement and zero-slope boundary conditions as follows:

$$
f_{n}(y)=a_{5} y^{5}+a_{4} y^{4}+a_{3} y^{3}+a_{2} y^{2}+a_{1} y+a_{0}
$$


where $f_{n}$ is the continuous flap deflection of flap $n, n=1,2,3, y$ is the BBL station, and $a_{i}, i=1,2,3,4,5$ are the polynomial coefficients determined by

$$
\left[\begin{array}{l}
a_{5} \\
a_{4} \\
a_{3} \\
a_{2} \\
a_{1} \\
a_{0}
\end{array}\right]=\left[\begin{array}{cccccc}
y_{1}^{5} & y_{1}^{4} & y_{1}^{3} & y_{1}^{2} & y_{1} & 1 \\
5 y_{1}^{4} & 4 y_{1}^{3} & 3 y_{1}^{2} & 2 y_{1} & 1 & 0 \\
y_{2}^{5} & y_{2}^{4} & y_{2}^{3} & y_{2}^{2} & y_{2} & 1 \\
5 y_{2}^{4} & 4 y^{3} & 3 y_{2}^{2} & 2 y_{2} & 1 & 0 \\
y_{3}^{5} & y_{3}^{4} & y_{3}^{3} & y_{3}^{2} & y_{3} & 1 \\
5 y_{3}^{4} & 4 y_{3}^{3} & 3 y_{3}^{2} & 2 y_{3} & 1 & 0
\end{array}\right]^{-1}\left[\begin{array}{c}
0 \\
0 \\
\frac{n f_{c}}{3} \\
0 \\
0 \\
0
\end{array}\right]=n f_{c}\left[\begin{array}{c}
-1.2929 \times 10^{-7} \\
2.2291 \times 10^{-5} \\
-4.5450 \times 10^{-4} \\
3.4045 \times 10^{-2} \\
-2.8738 \times 10^{-1} \\
7.6635 \times 10^{-1}
\end{array}\right]
$$

Figure 8 shows the BBL stations of the 12 flap segments. Aerodynamic vortex-lattice calculations show that VCCTE flap concept could produce a drag reduction benefits ranging from $46 \%$ to $66 \%$ at cruise.

The ESAC with an optimized wing shape equipped with the VCCTE flap system is illustrated in Figure 9.

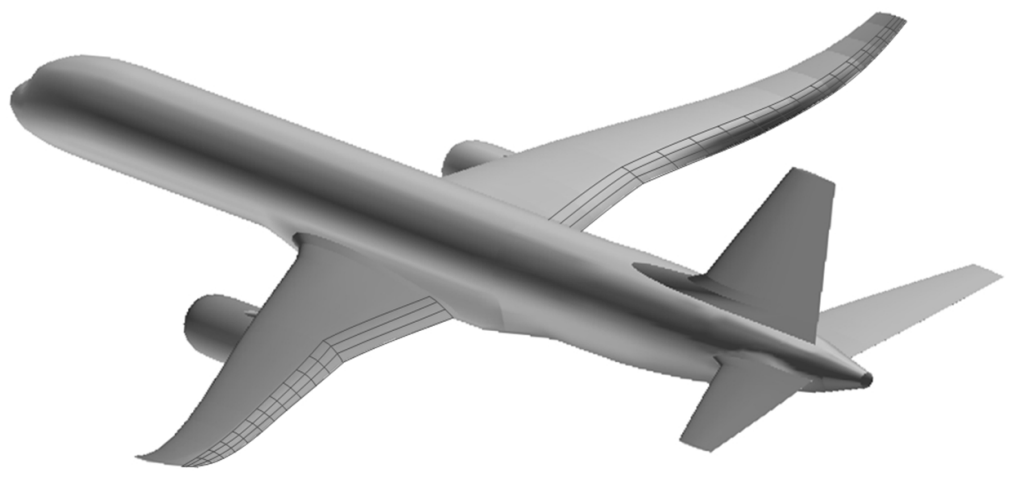

Figure 9 - Elastically Shaped Aircraft Concept with Variable Camber Continuous Trailing Edge Flap

\section{Nonlinear Aeroelastic Flight Dynamic Modeling}

To develop an understanding of the aeroelastic effects on aerodynamics and wing shaping control, a coupled aeroelastic flight dynamic model is developed for the wing structure which is modeled as beam-rod finite elements. The nonlinear model includes the propulsive effects of engine mass and thrust-induced stiffness as well as the fuel usage management to account for mass variation during cruise.

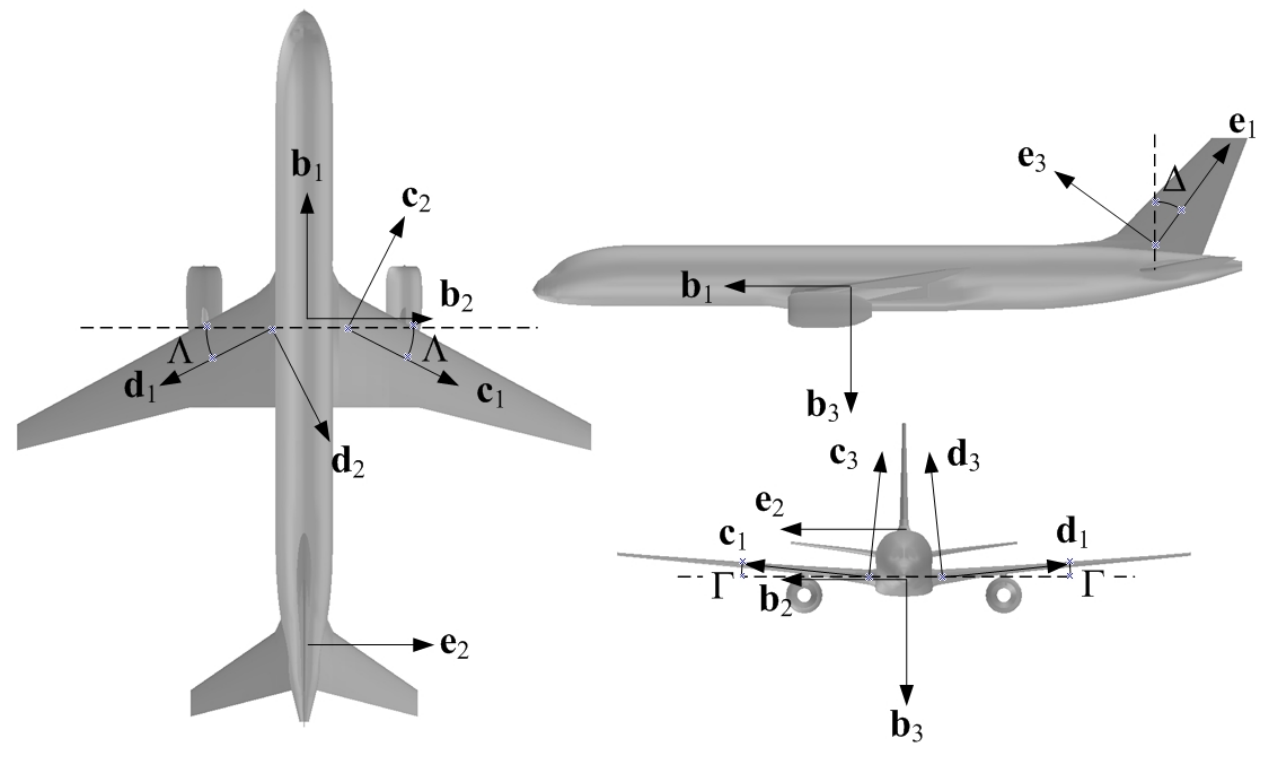

Figure 10 - Aircraft Reference Frames 


\section{A. Kinematic Analysis and Aeroelastic Angle of Attack}

Figure 10 illustrates three orthogonal views of a typical aircraft. Several reference frames are introduced to facilitate the rigid-body dynamic and structural dynamic analysis of the lifting surfaces. For example, the aircraft inertial reference frame $\mathrm{A}$ is defined by unit vectors $\mathbf{a}_{1}, \mathbf{a}_{2}$, and $\mathbf{a}_{3}$ fixed to the non-rotating earth. The aircraft body-fixed reference frame B is defined by unit vectors $\mathbf{b}_{1}, \mathbf{b}_{2}$, and $\mathbf{b}_{3}$ aligned with the roll, pitch, and yaw axes, respectively. The left wing elastic reference frame $D$ is defined by unit vectors $\mathbf{d}_{1}, \mathbf{d}_{2}$, and $\mathbf{d}_{3}$. The reference frames $B$ and $D$ are related by three successive rotations: 1) the first rotation about $-\mathbf{b}_{3}$ by the elastic axis sweep angle $\frac{\pi}{2}+\Lambda$ that results in an intermediate reference frame $\mathrm{B}^{\prime \prime}$ defined by unit vectors $\mathbf{b}_{1}^{\prime \prime}, \mathbf{b}_{2}^{\prime \prime}$, and $\mathbf{b}_{3}^{\prime \prime}$ (not shown), 2) the second rotation about $\mathbf{b}_{2}^{\prime \prime}$ by the elastic axis dihedral angle $\Gamma$ that results in an intermediate reference frame $\mathrm{D}^{\prime}$ defined by unit vectors $\mathbf{d}_{1}^{\prime}, \mathbf{d}_{2}^{\prime}$, and $\mathbf{d}_{3}^{\prime}$ (not shown), and 3) the third rotation about $\mathbf{d}_{1}^{\prime}$ by an angle $\pi$ that results in the reference frame D. This relationship is expressed as

$$
\begin{aligned}
{\left[\begin{array}{l}
\mathbf{b}_{1} \\
\mathbf{b}_{2} \\
\mathbf{b}_{3}
\end{array}\right]=} & {\left[\begin{array}{ccc}
-\sin \Lambda & \cos \Lambda & 0 \\
-\cos \Lambda & -\sin \Lambda & 0 \\
0 & 0 & 1
\end{array}\right]\left[\begin{array}{ccc}
\cos \Gamma & 0 & \sin \Gamma \\
0 & 1 & 0 \\
-\sin \Gamma & 0 & \cos \Gamma
\end{array}\right]\left[\begin{array}{ccc}
1 & 0 & 0 \\
0 & -1 & 0 \\
0 & 0 & -1
\end{array}\right]\left[\begin{array}{l}
\mathbf{d}_{1} \\
\mathbf{d}_{2} \\
\mathbf{d}_{3}
\end{array}\right] } \\
= & {\left[\begin{array}{ccc}
-\sin \Lambda \cos \Gamma & -\cos \Lambda & \sin \Lambda \sin \Gamma \\
-\cos \Lambda \cos \Gamma & \sin \Lambda & \cos \Lambda \sin \Gamma \\
-\sin \Gamma & 0 & -\cos \Gamma
\end{array}\right]\left[\begin{array}{l}
\mathbf{d}_{1} \\
\mathbf{d}_{2} \\
\mathbf{d}_{3}
\end{array}\right] }
\end{aligned}
$$

The aircraft velocity at the aircraft C.G. in the aircraft body-fixed reference B is expressed in the reference frame $\mathrm{D}$ as

$$
\begin{aligned}
& \mathbf{v}=\left[\begin{array}{lll}
u & v & w
\end{array}\right]\left[\begin{array}{ccc}
-\sin \Lambda \cos \Gamma & -\cos \Lambda & \sin \Lambda \sin \Gamma \\
-\cos \Lambda \cos \Gamma & \sin \Lambda & \cos \Lambda \sin \Gamma \\
-\sin \Gamma & 0 & -\cos \Gamma
\end{array}\right]\left[\begin{array}{l}
\mathbf{d}_{1} \\
\mathbf{d}_{2} \\
\mathbf{d}_{3}
\end{array}\right] \\
& =(-u \sin \Lambda \cos \Gamma-v \cos \Lambda \cos \Gamma-w \sin \Gamma) \mathbf{d}_{1}+(-u \cos \Lambda+v \sin \Lambda) \mathbf{d}_{2}+(u \sin \Lambda \sin \Gamma+v \cos \Lambda \sin \Gamma-w \cos \Gamma) \mathbf{d}_{3}
\end{aligned}
$$

where $(u, v, w)$ are the aircraft velocity components in the forward, lateral, and downward directions defined by the unit vectors $\left(\mathbf{b}_{1}, \mathbf{b}_{2}, \mathbf{b}_{3}\right)$, respectively.

The right wing reference frame $\mathrm{C}$ can be established in a similar manner. In the analysis, the aeroelastic effects on the fuselage, horizontal stabilizers, and vertical stabilizer are not considered, but the analytical method can be formulated for analyzing these lifting surfaces if necessary.

Consider an airfoil section on the left wing as shown in Figure 11 undergoing bending and twist deflections.

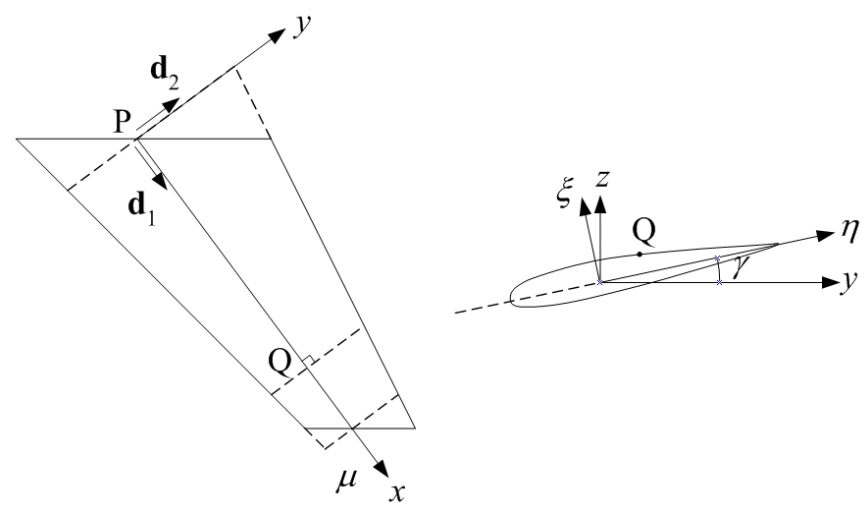

Figure 11 - Left Wing Reference Frame

Let $(x, y, z)$ be the coordinates of point $\mathrm{Q}$ on a wing airfoil section. The axial or extensional deflection of a wing is generally very small and therefore can usually be neglected. Let $\Theta$ be a torsional twist angle about the $x$-axis, positive 
nose-down, and let $W$ and $V$ be flapwise and chordwise bending deflections of point $\mathrm{Q}$, respectively. Then, the rotation angle due to the elastic deformation can be expressed as

$$
\phi(x, t)=\Theta \mathbf{d}_{1}-W_{x} \mathbf{d}_{2}+V_{x} \mathbf{d}_{3}
$$

where the subscripts $x$ and $t$ denote the partial derivatives of $\Theta, W$, and $V$.

Let $\left(x_{1}, y_{1}, z_{1}\right)$ be the coordinates of point $\mathbf{Q}$ on the airfoil in the reference frame $\mathbf{D}$. Then the coordinates $\left(x_{1}, y_{1}, z_{1}\right)$ are computed using the small angle approximation as

$$
\left[\begin{array}{c}
x_{1}(x, t) \\
y_{1}(x, t) \\
z_{1}(x, t)
\end{array}\right]=\left[\begin{array}{c}
x \\
y+V \\
z+W
\end{array}\right]+\left[\begin{array}{c}
\phi \times\left(y \mathbf{d}_{2}+z \mathbf{d}_{3}\right) \cdot \mathbf{d}_{1} \\
\phi \times\left(y \mathbf{d}_{2}+z \mathbf{d}_{3}\right) \cdot \mathbf{d}_{2} \\
\phi \times\left(y \mathbf{d}_{2}+z \mathbf{d}_{3}\right) \cdot \mathbf{d}_{3}
\end{array}\right]=\left[\begin{array}{c}
x-y V_{x}-z W_{x} \\
y+V-z \Theta \\
z+W+y \Theta
\end{array}\right]
$$

The relative velocity of the air approaching a wing section includes the contribution from the wing elastic deflection that results in changes in the local angle of attack. Since aerodynamic forces and moments are dependent on the local angle of attack, the wing aeroelastic deflections will generate additional elastic forces and moments. The local angle of attack depends on the relative approaching air velocity as well as the rotation angle $\phi$ from Eq. (10). The relative air velocity in turn also depends on the deflection-induced velocity. The velocity at point $\mathrm{Q}$ due to the aircraft velocity and angular velocity in the reference frame $\mathrm{D}$ is computed as

$$
\begin{aligned}
& \mathbf{v}_{Q}=\overline{\mathbf{v}}+\omega \times \mathbf{r}=\left(u \mathbf{b}_{1}+\right.\left.v \mathbf{b}_{2}+w \mathbf{b}_{3}\right)+\left(p \mathbf{b}_{1}+q \mathbf{b}_{2}+r \mathbf{b}_{3}\right) \times\left(-x_{a} \mathbf{b}_{1}-y_{a} \mathbf{b}_{2}-z_{a} \mathbf{b}_{3}\right) \\
&=\left(u+r y_{a}-q z_{a}\right) \mathbf{b}_{1}+\left(v-r x_{a}+p z_{a}\right) \mathbf{b}_{2}+\left(w+q x_{a}-p y_{a}\right) \mathbf{b}_{3} \\
&=\left[-\left(u+r y_{a}-q z_{a}\right) \sin \Lambda \cos \Gamma-\left(v-r x_{a}+p z_{a}\right) \cos \Lambda \cos \Gamma-\left(w+q x_{a}-p y_{a}\right) \sin \Gamma\right] \mathbf{d}_{1} \\
& \quad+\left[-\left(u+r y_{a}-q z_{a}\right) \cos \Lambda+\left(v-r x_{a}+p z_{a}\right) \sin \Lambda\right] \mathbf{d}_{2} \\
&+\left[\left(u+r y_{a}-q z_{a}\right) \sin \Lambda \sin \Gamma+\left(v-r x_{a}+p z_{a}\right) \cos \Lambda \sin \Gamma-\left(w+q x_{a}-p y_{a}\right) \cos \Gamma\right] \mathbf{d}_{3}
\end{aligned}
$$

where $(p, q, r)$ are aircraft angular velocity components in the roll, pitch, and yaw axes, and $\left(x_{a}, y_{a}, z_{a}\right)$ is the coordinate of point $\mathrm{Q}$ which may be taken as the aerodynamic center of a wing section in the aircraft body-fixed reference frame B relative to the aircraft C.G. (center of gravity) such that $x_{a}$ is positive when point Q is aft of the aircraft C.G., $y_{a}$ is positive when point $\mathrm{Q}$ is toward the left wing from the aircraft C.G., and $z_{a}$ is positive when point $\mathrm{Q}$ is above the aircraft C.G.

The local velocity at point $\mathrm{Q}$ due to aircraft rigid-body dynamics and aeroelastic deflections in the reference frame D is obtained as ${ }^{6}$

$$
\mathbf{v}=\mathbf{v}_{Q}+\dot{\phi} \times \Delta \mathbf{r}=v_{x} \mathbf{d}_{1}+v_{y} \mathbf{d}_{2}+v_{z} \mathbf{d}_{3}
$$

where

$$
\begin{aligned}
& {\left[\begin{array}{l}
v_{x} \\
v_{y} \\
v_{z}
\end{array}\right]=} \\
& {\left[\begin{array}{c}
-\left(u-q z_{a}\right) \sin \Lambda \cos \Gamma-\left(w+q x_{a}\right) \sin \Gamma-(z+W+y \Theta) W_{x t} \\
-\left(u+r y_{a}-q z_{a}\right) \cos \Lambda+\left(v-r x_{a}+p z_{a}\right) \sin \Lambda-(z+W+y \Theta) \Theta_{t} \\
\left(u+r y_{a}-q z_{a}\right) \sin \Lambda \sin \Gamma+\left(v-r x_{a}+p z_{a}\right) \cos \Lambda \sin \Gamma-\left(w+q x_{a}-p y_{a}\right) \cos \Gamma+W_{t}-z W_{x} W_{x t}+(y-z \Theta) \Theta_{t}
\end{array}\right]}
\end{aligned}
$$

A recent study based on this formulation has developed an aeroelastic model for a general aircraft motion in all three stability axes. ${ }^{7}$ For wing shaping control, the aircraft is assumed to be in a level flight with only variations in $\alpha$ and $q$. Assuming a small dihedral angle, then

$$
\left[\begin{array}{c}
v_{x} \\
v_{y} \\
v_{z}
\end{array}\right]=\left[\begin{array}{c}
-\left(u-q z_{a}\right) \sin \Lambda-\left(w+q x_{a}\right) \Gamma-(-z+W+y \Theta) W_{x t} \\
-\left(u-q z_{a}\right) \cos \Lambda-(-z+W+y \Theta) \Theta_{t} \\
\left(u-q z_{a}\right) \sin \Lambda \Gamma-\left(w+q x_{a}\right)+W_{t}-z W_{x} W_{x t}+(y-z \Theta) \Theta_{t}
\end{array}\right]
$$

To compute the aeroelastic forces and moments, the velocity must be transformed from the reference frame $\mathrm{D}$ to the airfoil local coordinate reference frame defined by $(\mu, \eta, \xi)$ as shown in Figure 10. Then the transformation can 
be performed using two successive rotation matrix multiplication operations as

$$
\begin{aligned}
{\left[\begin{array}{l}
v_{\mu} \\
v_{\eta} \\
v_{\xi}
\end{array}\right]=} & {\left[\begin{array}{ccc}
1 & 0 & 0 \\
0 & \cos (\Theta+\gamma) & \sin (\Theta+\gamma) \\
0 & -\sin (\Theta+\gamma) & \cos (\Theta+\gamma)
\end{array}\right]\left[\begin{array}{ccc}
\cos W_{x} & 0 & \sin W_{x} \\
0 & 1 & 0 \\
-\sin W_{x} & 0 & \cos W_{x}
\end{array}\right]\left[\begin{array}{l}
v_{x} \\
v_{y} \\
v_{z}
\end{array}\right] } \\
= & {\left[\begin{array}{c}
v_{x} \cos W_{x}+v_{z} \sin W_{x} \\
-v_{x} \sin W_{x} \sin (\Theta+\gamma)+v_{y} \cos (\Theta+\gamma)+v_{z} \cos W_{x} \sin (\Theta+\gamma) \\
-v_{x} \sin W_{x} \cos (\Theta+\gamma)-v_{y} \sin (\Theta+\gamma)+v_{z} \cos W_{x} \cos (\Theta+\gamma)
\end{array}\right] }
\end{aligned}
$$

For small deflections, the local velocity components can be simplified as

$$
\left[\begin{array}{c}
v_{\mu} \\
v_{\eta} \\
v_{\xi}
\end{array}\right]=\left[\begin{array}{c}
v_{x}+v_{z} W_{x} \\
-v_{x} W_{x}(\Theta+\gamma)+v_{y}+v_{z}(\Theta+\gamma) \\
v_{z}-v_{x} W_{x}-v_{y}(\Theta+\gamma)
\end{array}\right]
$$

Referring to Figure 12, the local aeroelastic angle of attack on the airfoil section due to the velocity components $v_{\eta}$ and $v_{\xi}$ in the reference frame $\mathrm{D}$ is computed as

$$
\alpha_{c}=\frac{v_{\xi}+w_{i}}{v_{\eta}}=\frac{\bar{v}_{\xi}+\Delta v_{\xi}+w_{i}}{\bar{v}_{\eta}+\Delta v_{\eta}}
$$

where $w_{i}$ is the downwash due to three-dimensional lift distribution over a finite wing and

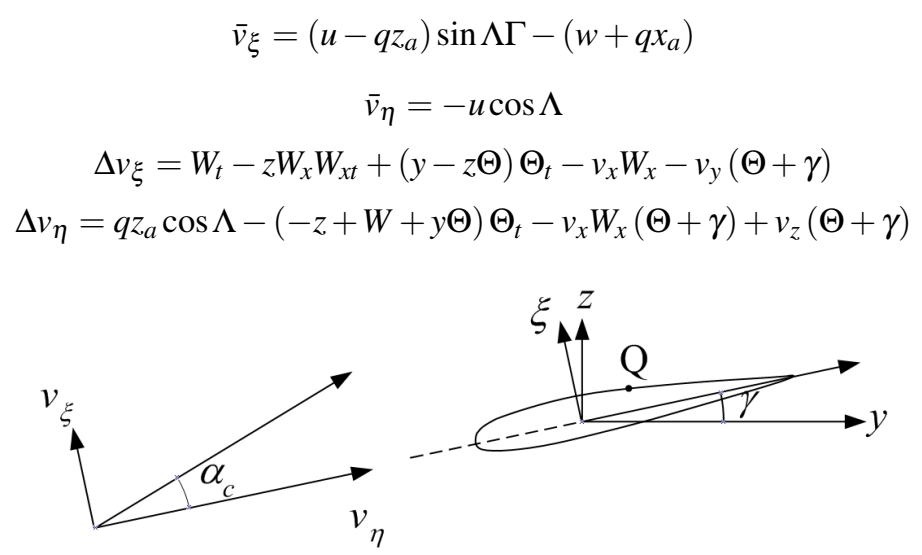

Figure 12 - Airfoil Local Coordinates

Then the local aeroelastic angle of attack can be evaluated as

$$
\begin{aligned}
\alpha_{c}(x, y, z)= & \frac{v_{\xi}+w_{i}}{\bar{v}_{\eta}}-\frac{\left(\bar{v}_{\xi}+w_{i}\right) \Delta v_{\eta}}{\bar{v}_{\eta}^{2}} \\
& =\frac{\left(u-q z_{a}\right) \sin \Lambda \Gamma-\left(w+q x_{a}\right)+W_{t}-z W_{x} W_{x t}+(y-z \Theta) \Theta_{t}-v_{x} W_{x}-v_{y}(\Theta+\gamma)+w_{i}}{-u \cos \Lambda} \\
& -\frac{\left[\left(u-q z_{a}\right) \sin \Lambda \Gamma-\left(w+q x_{a}\right)+w_{i}\right]\left[q z_{a} \cos \Lambda-(-z+W+y \Theta) \Theta_{t}-v_{x} W_{x}(\Theta+\gamma)+v_{z}(\Theta+\gamma)\right]}{u^{2} \cos ^{2} \Lambda}
\end{aligned}
$$

Expressing in terms of partial derivatives, then

$$
\begin{aligned}
\alpha_{c}(x, y, z)=\alpha_{0}+\frac{\partial \alpha_{c}}{\partial \alpha} \alpha & +\frac{\partial \alpha_{c}}{\partial q} q+\frac{\partial \alpha_{c}}{\partial \alpha^{2}} \alpha^{2}+\frac{\partial \alpha_{c}}{\partial \alpha q} \alpha q+\frac{\partial \alpha_{c}}{\partial q^{2}} q^{2}+\frac{\partial \alpha_{c}}{\partial W_{x}} W_{x}+\frac{\partial \alpha_{c}}{\partial W_{t}} W_{t}+\frac{\partial \alpha_{c}}{\partial \Theta} \Theta+\frac{\partial \alpha_{c}}{\partial \Theta_{t}} \Theta_{t} \\
+\frac{\partial \alpha_{c}}{\partial W \Theta_{t}} W \Theta_{t} & +\frac{\partial \alpha_{c}}{\partial W_{x} W_{x t}} W_{x} W_{x t}+\frac{\partial \alpha_{c}}{\partial \Theta W_{x}} \Theta W_{x}+\frac{\partial \alpha_{c}}{\partial \Theta W_{t}} \Theta W_{t}+\frac{\partial \alpha_{c}}{\partial \Theta \Theta_{t}} \Theta \Theta_{t}+\frac{\partial \alpha_{c}}{\partial W W_{x} W_{x t}} W W_{x} W_{x t} \\
& +\frac{\partial \alpha_{c}}{\partial \Theta W_{x} W_{x t}} \Theta W_{x} W_{x t}+\frac{\partial \alpha_{c}}{\partial \Theta^{2} \Theta_{t}} \Theta^{2} \Theta_{t}+\frac{\partial \alpha_{c}}{\partial \Theta W W_{x} W_{x t}} \Theta W W_{x} W_{x t}+\frac{\partial \alpha_{c}}{\partial \Theta^{2} W_{x} W_{x t}} \Theta^{2} W_{x} W_{x t}
\end{aligned}
$$


where the partial derivatives are given by

$$
\begin{gathered}
\alpha_{0}=-\gamma-\tan \Lambda \Gamma(1+\tan \Lambda \Gamma \gamma)-\frac{\alpha_{i}(1+\tan \Lambda \Gamma \gamma)}{\cos \Lambda} \\
\frac{\partial \alpha_{c}}{\partial \alpha}=\frac{1+2 \tan \Lambda \Gamma \gamma}{\cos \Lambda}+\frac{\alpha_{i} \gamma}{\cos ^{2} \Lambda} \\
\frac{\partial \alpha_{c}}{\partial q}=\frac{x_{a}(1+2 \tan \Lambda \Gamma \gamma)-\alpha_{i} z_{a}}{V_{\infty} \cos \Lambda}+\frac{z_{a} \gamma\left(1+2 \tan ^{2} \Lambda \Gamma^{2}\right)}{V_{\infty}}+\frac{\alpha_{i}\left(x_{a}+z_{a} \sin \Lambda \Gamma\right) \gamma}{V_{\infty} \cos ^{2} \Lambda} \\
\frac{\partial \alpha_{c}}{\partial \alpha^{2}}=-\frac{\gamma}{\cos ^{2} \Lambda} \\
\frac{\partial \alpha_{c}}{\partial \alpha q}=-\frac{2 x_{a} \gamma}{V_{\infty} \cos ^{2} \Lambda}+\frac{z_{a}(1-2 \tan \Lambda \Gamma \gamma)}{V_{\infty} \cos \Lambda} \\
\frac{\partial \alpha_{c}}{\partial q^{2}}=-\frac{x_{a}^{2} \gamma}{V_{\infty}^{2} \cos ^{2} \Lambda}+\frac{z_{a}^{2} \tan \Lambda \Gamma(1-\tan \Lambda \Gamma \gamma)}{V_{\infty}^{2}}+\frac{x_{a} z_{a}(1-2 \tan \Lambda \Gamma \gamma)}{V_{\infty}^{2} \cos \Lambda}
\end{gathered}
$$

$$
\begin{gathered}
\frac{\partial \alpha_{c}}{\partial W_{x}}=-\frac{\left(V_{\infty}-q z_{a}\right) \sin \Lambda+\left(V_{\infty} \alpha+q x_{a}\right) \Gamma}{V_{\infty} \cos \Lambda} \\
-\frac{\left[\left(V_{\infty}-q z_{a}\right) \sin \Lambda \Gamma-\left(V_{\infty} \alpha+q x_{a}\right)\right]\left[\left(V_{\infty}-q z_{a}\right) \sin \Lambda+\left(V_{\infty} \alpha+q x_{a}\right) \Gamma\right] \gamma}{V_{\infty}^{2} \cos ^{2} \Lambda}-\frac{\alpha_{i}\left[\left(V_{\infty}-q z_{a}\right) \sin \Lambda+\left(V_{\infty} \alpha+q x_{a}\right) \Gamma\right] \gamma}{V_{\infty} \cos ^{2} \Lambda} \\
\frac{\partial \alpha_{c}}{\partial W_{t}}=-\frac{1}{V_{\infty} \cos \Lambda}-\frac{\left[\left(V_{\infty}-q z_{a}\right) \sin \Lambda \Gamma-\left(V_{\infty} \alpha+q x_{a}\right)\right] \gamma}{V_{\infty}^{2} \cos ^{2} \Lambda}-\frac{\alpha_{i} \gamma}{V_{\infty} \cos ^{2} \Lambda} \\
\frac{\partial \alpha_{c}}{\partial \Theta}=-1+\frac{q z_{a}}{V_{\infty}}-\frac{\left[\left(V_{\infty}-q z_{a}\right) \sin \Lambda \Gamma-\left(V_{\infty} \alpha+q x_{a}\right)\right]^{2}}{V_{\infty}^{2} \cos ^{2} \Lambda} \\
\frac{\partial \alpha_{c}}{\partial \Theta_{t}}=-\frac{y+z \gamma}{V_{\infty} \cos \Lambda}-\frac{(y \gamma+z)\left[\left(V_{\infty}-q z_{a}\right) \sin \Lambda \Gamma-\left(V_{\infty} \alpha+q x_{a}\right)\right]}{V_{\infty}^{2} \cos ^{2} \Lambda}-\frac{\alpha_{i}(y \gamma+z)}{V_{\infty} \cos ^{2} \Lambda} \\
\frac{\partial \alpha_{c}}{\partial W \Theta_{t}}=-\frac{\gamma}{V_{\infty} \cos \Lambda}+\frac{\left(V_{\infty}-q z_{a}\right) \sin \Lambda \Gamma-\left(V_{\infty} \alpha+q x_{a}\right)}{V_{\infty}^{2} \cos ^{2} \Lambda}+\frac{\alpha_{i}}{V_{\infty} \cos ^{2} \Lambda} \\
\frac{\partial \alpha_{c}}{\partial W_{x} W_{x t}}=-\frac{z}{V_{\infty} \cos \Lambda}-\frac{z \gamma\left[\left(V_{\infty}-q z_{a}\right) \sin \Lambda \Gamma-\left(V_{\infty} \alpha+q x_{a}\right)\right]}{V_{\infty}^{2} \cos ^{2} \Lambda}
\end{gathered}
$$

$$
\begin{gathered}
\frac{\partial \alpha_{c}}{\partial \Theta W_{x}}=-\frac{\left[\left(V_{\infty}-q z_{a}\right) \sin \Lambda \Gamma-\left(V_{\infty} \alpha+q x_{a}\right)\right]\left[\left(V_{\infty}-q z_{a}\right) \sin \Lambda+\left(V_{\infty} \alpha+q x_{a}\right) \Gamma\right]}{V_{\infty}^{2} \cos ^{2} \Lambda}-\frac{\alpha_{i}\left[\left(V_{\infty}-q z_{a}\right) \sin \Lambda+\left(V_{\infty} \alpha+q x_{a}\right) \Gamma\right]}{V_{\infty} \cos ^{2} \Lambda} \\
\frac{\partial \alpha_{c}}{\partial \Theta W_{t}}=-\frac{\left(V_{\infty}-q z_{a}\right) \sin \Lambda \Gamma-\left(V_{\infty} \alpha+q x_{a}\right)}{V_{\infty}^{2} \cos ^{2} \Lambda}-\frac{\alpha_{i}}{V_{\infty} \cos ^{2} \Lambda} \\
\frac{\partial \alpha_{c}}{\partial \Theta \Theta_{t}}=-\frac{y \gamma}{V_{\infty} \cos \Lambda}+\frac{z \gamma\left[\left(V_{\infty}-q z_{a}\right) \sin \Lambda \Gamma-\left(V_{\infty} \alpha+q x_{a}\right)\right]}{V_{\infty}^{2} \cos ^{2} \Lambda}+\frac{\alpha_{i} z \gamma}{V_{\infty} \cos ^{2} \Lambda} \\
\frac{\partial \alpha_{c}}{\partial W W_{x} W_{x t}}=-\frac{1}{V_{\infty} \cos \Lambda}-\frac{\left[\left(V_{\infty}-q z_{a}\right) \sin \Lambda \Gamma-\left(V_{\infty} \alpha+q x_{a}\right)\right] \gamma}{V_{\infty}^{2} \cos ^{2} \Lambda}-\frac{\alpha_{i} \gamma}{V_{\infty} \cos ^{2} \Lambda} \\
\frac{\partial \alpha_{c}}{\partial \Theta W_{x} W_{x t}}=-\frac{y}{V_{\infty} \cos \Lambda}-\frac{(y \gamma+z)\left[\left(V_{\infty}-q z_{a}\right) \sin \Lambda \Gamma-\left(V_{\infty} \alpha+q x_{a}\right)\right]}{V_{\infty}^{2} \cos ^{2} \Lambda}-\frac{\alpha_{i} y \gamma}{V_{\infty} \cos ^{2} \Lambda} \\
\frac{\partial \alpha_{c}}{\partial \Theta^{2} \Theta_{t}}=-\frac{y}{V_{\infty} \cos \Lambda}+\frac{z\left[\left(V_{\infty}-q z_{a}\right) \sin \Lambda \Gamma-\left(V_{\infty} \alpha+q x_{a}\right)\right]}{V_{\infty}^{2} \cos ^{2} \Lambda}+\frac{\alpha_{i} z}{V_{\infty} \cos ^{2} \Lambda}
\end{gathered}
$$




$$
\begin{gathered}
\frac{\partial \alpha_{c}}{\partial \Theta W W_{x} W_{x t}}=-\frac{\left[\left(V_{\infty}-q z_{a}\right) \sin \Lambda \Gamma-\left(V_{\infty} \alpha+q x_{a}\right)\right]}{V_{\infty}^{2} \cos ^{2} \Lambda}-\frac{\alpha_{i}}{V_{\infty} \cos ^{2} \Lambda} \\
\frac{\partial \alpha_{c}}{\partial \Theta^{2} W_{x} W_{x t}}=-\frac{y\left[\left(V_{\infty}-q z_{a}\right) \sin \Lambda \Gamma-\left(V_{\infty} \alpha+q x_{a}\right)\right]}{V_{\infty}^{2} \cos ^{2} \Lambda}-\frac{\alpha_{i} y}{V_{\infty} \cos ^{2} \Lambda}
\end{gathered}
$$

Let point $\mathrm{Q}$ be the aerodynamic center, then the local angle of attack of an airfoil section is evaluated at $y=-e$ and $z=0$, where $e$ is the location of the aerodynamic center relative to the elastic axis, positive when the aerodynamic center is forward of the elastic axis. Let $\alpha_{r}$ be the rigid-body angle of attack and $\alpha_{e}$ be the aeroelastic angle of attack at the aerodynamic center. Then, the angle of attack at the aerodynamic center, $\alpha_{a c}$, is expressed as

$$
\alpha_{a c}(x)=\alpha_{c}(x,-e, 0)=\alpha_{r}+\alpha_{e}(x)
$$

where

$$
\begin{gathered}
\alpha_{r}=\alpha_{0}+\frac{\partial \alpha_{c}}{\partial \alpha} \alpha+\frac{\partial \alpha_{c}}{\partial q} q+\frac{\partial \alpha_{c}}{\partial \alpha^{2}} \alpha^{2}+\frac{\partial \alpha_{c}}{\partial \alpha q} \alpha q+\frac{\partial \alpha_{c}}{\partial q^{2}} q^{2} \\
\alpha_{e}=\frac{\partial \alpha_{c}}{\partial W_{x}} W_{x}+\frac{\partial \alpha_{c}}{\partial \Theta} \Theta+\frac{\partial \alpha_{c}}{\partial \Theta W_{x}} \Theta W_{x}+\left(\frac{\partial \alpha_{c}}{\partial W_{t}}+\frac{\partial \alpha_{c}}{\partial \Theta W_{t}} \Theta\right) W_{t}+\left(\frac{\partial \alpha_{c}}{\partial \Theta_{t}}+\frac{\partial \alpha_{c}}{\partial W \Theta_{t}} W+\frac{\partial \alpha_{c}}{\partial \Theta \Theta_{t}} \Theta+\frac{\partial \alpha_{c}}{\partial \Theta^{2} \Theta_{t}} \Theta^{2}\right) \Theta_{t} \\
+\left(\frac{\partial \alpha_{c}}{\partial W_{x} W_{x t}}+\frac{\partial \alpha_{c}}{\partial W W_{x} W_{x t}} W+\frac{\partial \alpha_{c}}{\partial \Theta W_{x} W_{x t}} \Theta+\frac{\partial \alpha_{c}}{\partial \Theta W W_{x} W_{x t}} \Theta W+\frac{\partial \alpha_{c}}{\partial \Theta^{2} W_{x} W_{x t}} \Theta^{2}\right) W_{x} W_{x t}
\end{gathered}
$$

The partial derivatives of $W$ and $\Theta$ with respect to time contribute to the aerodynamic damping and are evaluated at the aerodynamic center as

$$
\begin{gathered}
\frac{\partial \alpha_{c}}{\partial \Theta_{t}}=\frac{e}{V_{\infty} \cos \Lambda}+\frac{e \gamma\left[\left(V_{\infty}-q z_{a}\right) \sin \Lambda \Gamma-\left(V_{\infty} \alpha+q x_{a}\right)\right]}{V_{\infty}^{2} \cos ^{2} \Lambda}+\frac{\alpha_{i} e \gamma}{V_{\infty} \cos ^{2} \Lambda} \\
\frac{\partial \alpha_{c}}{\partial W_{x} W_{x t}}=0 \\
\frac{\partial \alpha_{c}}{\partial \Theta \Theta_{t}}=\frac{e \gamma}{V_{\infty} \cos \Lambda} \\
\frac{\partial \alpha_{c}}{\partial \Theta W_{x} W_{x t}}=\frac{e}{V_{\infty} \cos \Lambda}+\frac{e \gamma\left[\left(V_{\infty}-q z_{a}\right) \sin \Lambda \Gamma-\left(V_{\infty} \alpha+q x_{a}\right)\right]}{V_{\infty}^{2} \cos ^{2} \Lambda}+\frac{\alpha_{i} e \gamma}{V_{\infty} \cos ^{2} \Lambda} \\
\frac{\partial \alpha_{c}}{\partial \Theta^{2} \Theta_{t}}=\frac{e}{V_{\infty} \cos \Lambda} \\
\frac{\partial \alpha_{c}}{\partial \Theta^{2} W_{x} W_{x t}}=\frac{e\left[\left(V_{\infty}-q z_{a}\right) \sin \Lambda \Gamma-\left(V_{\infty} \alpha+q x_{a}\right)\right]}{V_{\infty}^{2} \cos ^{2} \Lambda}+\frac{\alpha_{i} e}{V_{\infty} \cos ^{2} \Lambda}
\end{gathered}
$$

Let point $\mathrm{Q}$ be the mid-chord location, then the local angle of attack of an airfoil is evaluated at $y=e_{m}$ and $z=0$, where $e_{m}$ is the distance of the mid-chord location relative to the elastic axis, positive when the mid-chord location is aft of the elastic axis. Let $\alpha_{m}$ be the aeroelastic angle of attack at the mid-chord location. Then, the angle of attack at the mid-chord location, $\alpha_{m c}$, is expressed as

$$
\alpha_{m c}=\alpha_{c}\left(x, e_{m}, 0\right)=\alpha_{r}+\alpha_{m}(x)
$$

\section{B. Forces and Moments and Aeroelastic Equations}

\section{Aerodynamic Forces and Moments}

The unsteady circulatory lift coefficient acting at the aerodynamic center is modeled using the Theodorsen's unsteady aerodynamics theory as follows: ${ }^{8}$

$$
c_{L_{a c}}(x)=c_{L_{\alpha}}(x) \alpha_{a c}(x) F(k)+c_{L_{\alpha}}(x) \frac{\dot{\alpha}_{a c}(x) c(x)}{2 V_{\infty}} \frac{G(k)}{k}+c_{L_{\delta}}(x) \delta(x)
$$

where $k=\frac{\omega \bar{c}}{2 V_{\infty}}$ is the reduced frequency parameter, $\omega$ is the frequency of wing oscillations, $\bar{c}$ is the mean aerodynamic chord, $c_{L_{0}}$ is the section lift coefficient at zero angle of attack, $c_{L_{\alpha}}$ is the section lift curve slope, $c_{L_{\delta}}$ is the section lift derivative with respect to the VCCTE flap deflection, $c$ is the section chord, and $\delta$ is the VCCTE flap deflection. 
The functions $F(k)$ and $G(k)$ are the real part and imaginary part of the Theodorsen's function $C(k)$ which is also expressed in terms of Hankel functions of the second kind $H_{n}^{(2)}(k)$ as

$$
C(k)=\frac{H_{1}^{(2)}(k)}{H_{1}^{(2)}(k)+i H_{0}^{(2)}(k)}=F(k)-i G(k)
$$

where $F(k)>0$ and $G(k)>0$.

When $k=0$, the airfoil motion is steady and $C(k)$ is real and unity. As $k$ increases, there is a phase lag introduced as the magnitude of $G(k)$ increases as shown in Figures 13 and 14. The limiting values of $F(k)$ and $G(k)$ are $1 / 2$ and 0 as $k \rightarrow \infty .{ }^{9}$

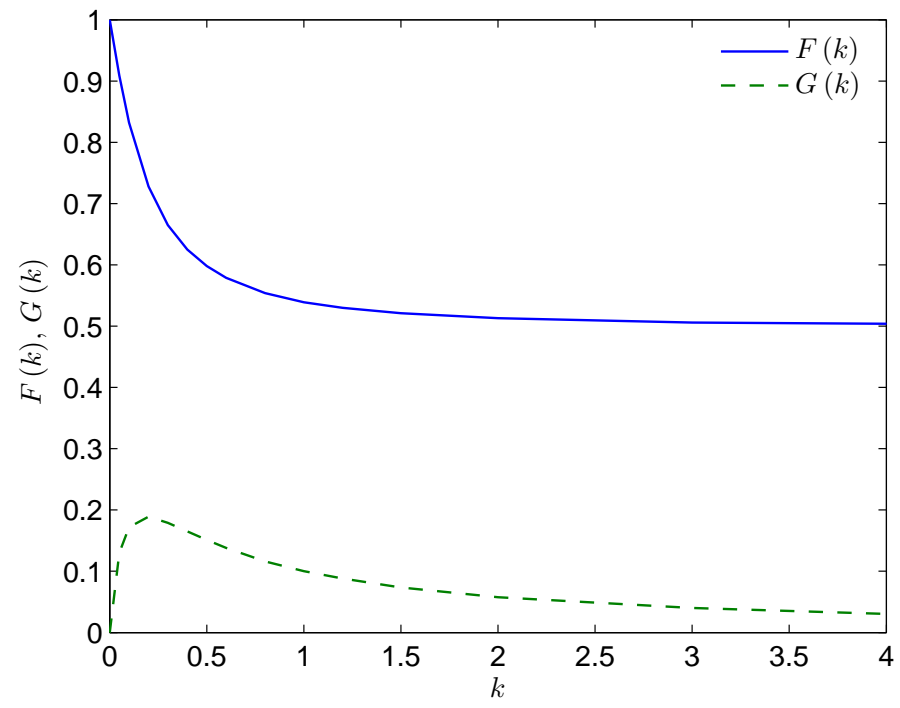

Figure 13 - Theodorsen's Function

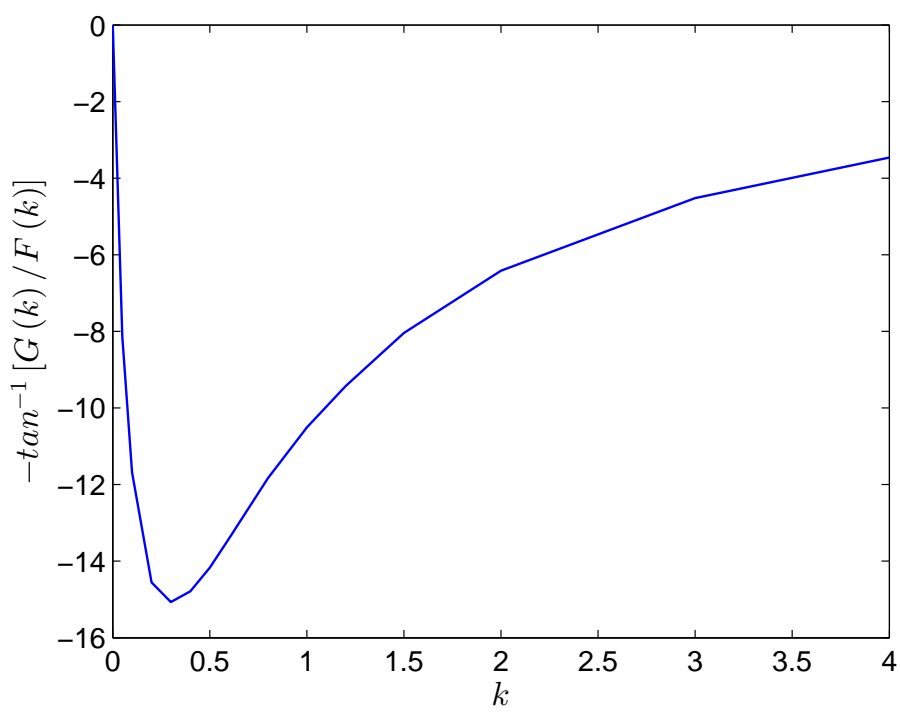

Figure 14 - Theodorsen's Function Phase lag

In addition, the apparent mass of the air contributes to the lift force acting at the mid-chord location as follows:

$$
c_{L_{m c}}(x)=\frac{\pi \dot{\alpha}_{m c}(x) c(x)}{2 V_{\infty}}
$$


The total lift coefficient is

$$
c_{L}(x)=c_{L_{a c}}(x)+c_{L_{m c}}(x)
$$

Separating the rigid-body and aeroelastic contributions from the total lift coefficient, one can write

$$
c_{L}(x)=c_{L_{r}}(x)+c_{L_{e}}(x)+\Delta c_{L_{\delta}}(x)
$$

where $c_{L_{r}}$ is the rigid-body lift coefficient, $c_{L_{e}}$ is the aeroelastic lift coefficient, and $\Delta c_{L_{\delta}}$ is the incremental lift coefficient due to the VCCTE flap deflection

$$
\begin{gathered}
c_{L_{r}}(x)=c_{L_{\alpha}}(x) \alpha_{r}(x) F(k)+\left[c_{L_{\alpha}}(x) \frac{G(k)}{k}+\pi\right] \frac{\dot{\alpha}_{r}(x) c(x)}{2 V_{\infty}} \\
c_{L_{e}}(x)=c_{L_{\alpha}}(x) \alpha_{e}(x) F(k)+c_{L_{\alpha}}(x) \frac{\dot{\alpha}_{e}(x) c(x)}{2 V_{\infty}} \frac{G(k)}{k}+\frac{\pi \dot{\alpha}_{m}(x) c(x)}{2 V_{\infty}} \\
\Delta c_{L_{\delta}}(x)=c_{L_{\delta}}(x) \delta(x)
\end{gathered}
$$

The drag coefficient is modeled using a parabolic drag polar as

$$
c_{D}(x)=c_{D_{0}}(x)+k(x) c_{L}^{2}(x)+c_{D_{\delta}}(x) \delta(x)+c_{D_{\delta^{2}}}(x) \delta^{2}(x)
$$

where $c_{D_{0}}$ is the section parasitic drag coefficient, $k$ is the section drag polar parameter, $c_{D_{\delta}}$ and $c_{D_{\delta^{2}}}$ are the section drag derivatives with respect to the VCCTE flap deflection.

One can write the drag coefficient in terms of the rigid-body and aeroelastic contributions as

$$
\begin{gathered}
c_{D}(x)=c_{D_{r}}(x)+c_{D_{e}}(x)+\Delta c_{D_{\delta}}(x) \\
c_{D_{r}}(x)=c_{D_{0}}(x)+k(x) c_{L_{r}}^{2}(x) \\
c_{D_{e}}(x)=k(x) c_{L_{e}}(x)\left[2 c_{L_{r}}(x)+c_{L_{e}}(x)\right] \\
\Delta c_{D_{\delta}}(x)=k(x) c_{L_{\delta}}(x) \delta(x)\left[2 c_{L_{r}}(x)+2 c_{L_{e}}(x)+c_{L_{\delta}}(x) \delta(x)\right]+c_{D_{\delta}}(x) \delta(x)+c_{D_{\delta^{2}}}(x) \delta^{2}(x)
\end{gathered}
$$

The pitching moment coefficient about the elastic axis is computed as

$$
c_{m}(x)=c_{m_{a c}}(x)+\frac{e(x)}{c(x)} c_{L_{a c}}(x)-\frac{e_{m}(x)}{c(x)} c_{L_{m c}}(x)+c_{m_{\delta}}(x) \delta(x)
$$

where $c_{m_{a c}}$ is the section pitching moment coefficient about the section aerodynamic center and $c_{m_{\delta}}$ is the section pitching moment derivative with respect to the VCCTE flap deflection.

The lift force, drag force, and pitching moment per unit span about the elastic axis are then expressed as

$$
\begin{gathered}
f_{z_{a}}=c_{L} q_{\infty} \cos ^{2} \Lambda c \\
f_{y_{a}}=c_{D} q_{\infty} \cos ^{2} \Lambda c \\
m_{x_{a}=}-c_{m} q_{\infty} \cos ^{2} \Lambda c^{2}
\end{gathered}
$$

\section{Propulsive Forces and Moments}

For wing-mounted engines, both the engine mass and thrust contribute to the wing aeroelasticity. ${ }^{7}$ The propulsive effects of the aircraft engines are therefore accounted for in the analysis. The propulsive force and moment vector are computed as

$$
\begin{gathered}
\mathbf{f}_{e}=\delta\left(x-x_{e}\right)\left[\begin{array}{ccc}
T & 0 & m_{e} g
\end{array}\right]\left[\begin{array}{ccc}
-\sin \Lambda & -\cos \Lambda & \Gamma \\
-\cos \Lambda & \sin \Lambda & \Gamma \\
-\Gamma & 0 & -1
\end{array}\right]\left[\begin{array}{l}
\mathbf{d}_{1} \\
\mathbf{d}_{2} \\
\mathbf{d}_{3}
\end{array}\right] \\
\mathbf{m}_{e}=\mathbf{r}_{e} \times \mathbf{f}_{e}=\left(x_{e} \mathbf{d}_{1}-y_{e} \mathbf{d}_{2}-z_{e} \mathbf{d}_{3}\right) \times \mathbf{f}_{e}
\end{gathered}
$$

where $T$ is the engine thrust, $m_{e}$ is the engine mass, $\left(x_{e}, y_{e}, z_{e}\right)$ is the coordinate of the engine thrust center such that $y_{e}$ is positive forward of the elastic axis and $z_{e}$ is positive below the elastic axis, and $\delta\left(x-x_{e}\right)$ is the Dirac delta function such that

$$
\int \delta\left(x-x_{e}\right) f(x) d x=f\left(x_{e}\right)
$$


Transforming into the local coordinate reference frame, the propulsive forces and moments are given by

$$
\begin{gathered}
f_{x_{e}}=\delta\left(x-x_{e}\right)\left[-T \sin \Lambda-m_{e} g \Gamma+\left(T \sin \Lambda \Gamma-m_{e} g\right) W_{x}\right] \\
f_{y_{e}}=\delta\left(x-x_{e}\right)\left[-T \cos \Lambda+\left(T \sin \Lambda \Gamma-m_{e} g\right)(\Theta+\gamma)+\left(T \sin \Lambda-m_{e} g \Gamma\right) W_{x}(\Theta+\gamma)\right] \\
f_{z_{e}}=\delta\left(x-x_{e}\right)\left[T \sin \Lambda \Gamma-m_{e} g+\left(T \sin \Lambda+m_{e} g \Gamma\right) W_{x}+T \cos \Lambda(\Theta+\gamma)\right] \\
m_{x_{e}}=\delta\left(x-x_{e}\right)\left[-T y_{e} \sin \Lambda \Gamma-T z_{e} \cos \Lambda+m_{e} g y_{e}-\left(T x_{e} \cos \Lambda+T y_{e} \sin \Lambda+m_{e} g y_{e} \Gamma\right) W_{x}\right] \\
m_{y_{e}}=\delta\left(x-x_{e}\right)\left[-T x_{e} \sin \Lambda \Gamma+T z_{e} \sin \Lambda+m_{e} g x_{e}+m_{e} g z_{e} \Gamma-\left(T x_{e} \cos \Lambda+T y_{e} \sin \Lambda+m_{e} g y_{e} \Gamma\right)(\Theta+\gamma)\right. \\
\left.-\left(-T y_{e} \sin \Lambda \Gamma-T z_{e} \cos \Lambda+m_{e} g y_{e}\right) W_{x}(\Theta+\gamma)\right] \\
m_{z_{e}}=\delta\left(x-x_{e}\right)\left[-T x_{e} \cos \Lambda-T y_{e} \sin \Lambda-m_{e} g y_{e} \Gamma-\left(-T y_{e} \sin \Lambda \Gamma-T z_{e} \cos \Lambda+m_{e} g y_{e}\right) W_{x}\right. \\
\left.-\left(-T x_{e} \sin \Lambda \Gamma+m_{e} g x_{e}+T z_{e} \sin \Lambda+m_{e} g z_{e} \Gamma\right)(\Theta+\gamma)\right]
\end{gathered}
$$

The partial derivatives of the moment components are

$$
\begin{gathered}
\frac{\partial m_{x_{e}}}{\partial x}=-\delta\left(x-x_{e}\right)\left(T x_{e} \cos \Lambda+T y_{e} \sin \Lambda+m_{e} g y_{e} \Gamma\right) W_{x x} \\
\frac{\partial m_{y_{e}}}{\partial x}=-\delta\left(x-x_{e}\right)\left\{\left(T x_{e} \cos \Lambda+T y_{e} \sin \Lambda+m_{e} g y_{e} \Gamma\right)\left(\Theta_{x}+\gamma^{\prime}\right)\right. \\
\left.+\left(-T y_{e} \sin \Lambda \Gamma-T z_{e} \cos \Lambda+m_{e} g y_{e}\right)\left[W_{x x}(\Theta+\gamma)+W_{x}\left(\Theta_{x}+\gamma^{\prime}\right)\right]\right\} \\
\frac{\partial m_{z_{e}}}{\partial x}=-\left(x-x_{e}\right)\left[\left(-T y_{e} \sin \Lambda \Gamma-T z_{e} \cos \Lambda+m_{e} g y_{e}\right) W_{x x}\right. \\
\left.+\left(-T x_{e} \sin \Lambda \Gamma+m_{e} g x_{e}+T z_{e} \sin \Lambda+m_{e} g z_{e} \Gamma\right)\left(\Theta_{x}+\gamma^{\prime}\right)\right]
\end{gathered}
$$

\section{Nonlinear Aeroelastic Equations}

The equilibrium conditions for aeroelastic wing bending and torsion are expressed as

$$
\begin{gathered}
\frac{\partial M_{x}}{\partial x}=-m_{x} \\
\frac{\partial^{2} M_{y}}{\partial x^{2}}=f_{z}-\frac{\partial m_{y}}{\partial x}
\end{gathered}
$$

where $m_{x}$ is the pitching moment per unit span about the elastic axis, $f_{z}$ is the vertical lift force per unit span, and $m_{y}$ is the bending moment per unit span about the flapwise axis. The moment equations are given by ${ }^{10}$

$$
\left[\begin{array}{c}
M_{x} \\
M_{y}
\end{array}\right]=\left[\begin{array}{cc}
G J+E B_{1}\left(\gamma^{\prime}\right)^{2} & -E B_{2} \gamma^{\prime} \\
-E B_{2} \gamma^{\prime} & E I_{y y}
\end{array}\right]\left[\begin{array}{c}
\Theta_{x} \\
W_{x x}
\end{array}\right]
$$

where $E$ is the Young's modulus; $G$ is the shear modulus; $\gamma^{\prime}$ is the derivative of the wing pre-twist angle; $I_{y y}$ is the section area moment of inertia about the flapwise axis; $J$ is the torsional constant; and $B_{1}$ and $B_{2}$ are the bending-torsion coupling constants which are defined as

$$
\left[\begin{array}{l}
B_{1} \\
B_{2}
\end{array}\right]=\iint\left(y^{2}+z^{2}\right)\left[\begin{array}{c}
y^{2}+z^{2} \\
z
\end{array}\right] d y d z
$$

For pre-twisted wings, the bending deflection is thus coupled to the torsional deflection due to the slope of the wing pre-twist angle. This coupling can be significant if the wash-out slope $\gamma^{\prime}$ is dominant. 
The aeroelastic equations that describe the coupled bending and torsion motion of the wing are then established as

$$
\begin{aligned}
& \begin{aligned}
& \frac{\partial^{2}}{\partial x^{2}}\left(-E B_{2} \gamma^{\prime} \Theta_{x}+E I_{y y} W_{x x}\right)= \\
&-m W_{t t}+ m e_{c g} \Theta_{t t}+\delta\left(x-x_{e}\right)\left(-m_{e} W_{t t}+m_{e} y_{e} \Theta_{t t}\right)-m g+c_{L} q_{\infty} \cos ^{2} \Lambda c \\
&+\delta\left(x-x_{e}\right)\left[T \sin \Lambda \Gamma-m_{e} g+\left(T \sin \Lambda+m_{e} g \Gamma\right) W_{x}+T \cos \Lambda(\Theta+\gamma)\right] \\
&+\delta\left(x-x_{e}\right)\left(T x_{e} \cos \Lambda+T y_{e} \sin \Lambda+m_{e} g y_{e} \Gamma\right)\left(\Theta_{x}+\gamma^{\prime}\right) \\
&+\delta\left(x-x_{e}\right)\left(-T y_{e} \sin \Lambda \Gamma-T z_{e} \cos \Lambda+m_{e} g y_{e}\right)\left[W_{x x}(\Theta+\gamma)+W_{x}\left(\Theta_{x}+\gamma^{\prime}\right)\right] \\
& \frac{\partial}{\partial x}\left\{\left[G J+E B_{1}\left(\gamma^{\prime}\right)^{2}\right] \Theta_{x}-E B_{2} \gamma^{\prime} W_{x x}\right\}= \\
& m k^{2} \Theta_{t t}-m e_{c g} W_{t t}+\delta\left(x-x_{e}\right)\left[m_{e}\left(y_{e}^{2}+z_{e}^{2}\right) \Theta_{t t}-m_{e} y_{e} W_{t t}\right]-m g e_{c g}+c_{m} q_{\infty} \cos ^{2} \Lambda c^{2} \\
&-\delta\left(x-x_{e}\right)\left[-T y_{e} \sin \Lambda \Gamma-T z_{e} \cos \Lambda+m_{e} g y_{e}-\left(T x_{e} \cos \Lambda+T y_{e} \sin \Lambda+m_{e} g y_{e} \Gamma\right) W_{x}\right]
\end{aligned}
\end{aligned}
$$

These equations are coupled with the rigid aircraft states $\alpha$ and $q$. To establish this coupling, consider the rigid aircraft longitudinal dynamics in the stability axes described by

$$
\begin{gathered}
m_{a} \dot{V}_{\infty}=-C_{D} q_{\infty} S+T \cos \alpha-m_{a} g \sin (\theta-\alpha) \\
m_{a} V_{\infty} \dot{\alpha}=-C_{L} q_{\infty} S-T \sin \alpha+m_{a} g \cos (\theta-\alpha) \\
\bar{I}_{y y} \dot{q}=C_{m} q_{\infty} S \bar{c}+\frac{T \bar{z}_{e}}{q_{\infty} S \bar{c}} \\
\dot{\theta}=q
\end{gathered}
$$

where $m_{a}$ is the aircraft mass, $\theta$ is the pitch attitude, $S$ is the aircraft reference wing area, $\bar{I}_{y y}$ is the aircraft principal moment of inertia about the pitch axis, and $\bar{z}_{e}$ is the offset of the thrust line below the aircraft CG.

The aerodynamic coefficients now include the contributions from the aeroelasticity. The aircraft lift coefficient for symmetric flight can be expressed as

$$
C_{L}=C_{L_{a}}+C_{L_{e}}+\Delta C_{L_{\delta}}
$$

where $C_{L_{a}}$ is the aircraft lift coefficient due to rigid-body aircraft, $C_{L_{e}}$ is the aircraft lift coefficient due to wing aeroelasticity, and $\Delta C_{L_{\delta}}$ is the aircraft incremental lift coefficient due to the VCCTE flap deflection given by

$$
\begin{gathered}
C_{L_{a}}=C_{L_{0}}+C_{L_{\alpha}} \alpha+C_{L_{\dot{\alpha}}} \frac{\dot{\alpha} \bar{c}}{2 \bar{V}_{\infty}}+C_{L_{q}} \frac{q \bar{c}}{2 \bar{V}_{\infty}}+C_{L_{u}}\left(\frac{V_{\infty}}{\bar{V}_{\infty}}-1\right)+C_{L_{\alpha^{2}}} \alpha^{2}+C_{L_{\alpha q}} \frac{\alpha q \bar{c}}{2 \bar{V}_{\infty}}+C_{L_{q^{2}}}\left(\frac{q \bar{c}}{2 \bar{V}_{\infty}}\right)^{2}+C_{L_{\delta_{e}}} \delta_{e} \\
C_{L_{e}}=\frac{2}{S} \int_{0}^{L} c_{L_{e}} \cos ^{2} \Lambda c d x \\
\Delta C_{L_{\delta}}=\frac{2}{S} \int_{0}^{L} \Delta c_{L_{\delta}} \cos ^{2} \Lambda c d x
\end{gathered}
$$

where $\bar{c}$ is the mean aerodynamic chord and $\bar{V}_{\infty}$ is the trim airspeed.

The aircraft drag coefficient may be modeled by a parabolic drag polar as

$$
C_{D}=C_{D_{a}}+C_{D_{e}}+\Delta C_{D_{\delta}}
$$

where $C_{D_{a}}$ is the aircraft drag coefficient due to rigid-body aircraft, $C_{D_{e}}$ is the aircraft drag coefficient due to wing aeroelasticity, and $\Delta C_{D_{\delta}}$ is the aircraft incremental drag coefficient due to the VCCTE flap deflection given by

$$
\begin{gathered}
C_{D_{a}}=C_{D_{0}}+\frac{C_{L_{a}}^{2}}{\pi A R \varepsilon} \\
C_{D_{e}}=\frac{2}{S} \int_{0}^{L} c_{D_{e}} \cos ^{2} \Lambda c d x
\end{gathered}
$$




$$
\Delta C_{D_{\delta}}=\frac{2}{S} \int_{0}^{L} \Delta c_{D_{\delta}} \cos ^{2} \Lambda c d x
$$

where $A R$ is the wing aspect ratio and $\varepsilon$ is the span efficiency factor.

The pitching moment coefficient of an aircraft is also influenced by the aeroelastic effects due to changes in wing lift characteristics as

$$
C_{m}=C_{m_{a}}+C_{m_{e}}+\Delta C_{m_{\delta}}
$$

where $C_{m_{a}}$ is the aircraft pitching coefficient due to rigid-body aircraft, $C_{m_{e}}$ is the aircraft pitching moment coefficient due to wing aeroelasticity, $\Delta C_{m_{\delta}}$ is the aircraft incremental pitching moment coefficient due to the VCCTE flap deflection given by

$$
\begin{gathered}
C_{m_{a}}=C_{m_{0}}+C_{m_{\alpha}} \alpha+C_{m_{\dot{\alpha}}} \frac{\dot{\alpha} \bar{c}}{2 \bar{V}_{\infty}}+C_{m_{q}} \frac{q \bar{c}}{2 \bar{V}_{\infty}}+C_{m_{u}}\left(\frac{V_{\infty}}{\bar{V}_{\infty}}-1\right)+C_{m_{\alpha^{2}}} \alpha^{2}+C_{m_{\alpha q}} \frac{\alpha q \bar{c}}{2 \bar{V}_{\infty}}+C_{m_{q^{2}}}\left(\frac{q \bar{c}}{2 \bar{V}_{\infty}}\right)^{2}+C_{m_{\delta_{e}}} \delta_{e} \\
C_{m_{e}}=-\frac{2}{S} \int_{0}^{L}\left(c_{L_{e}} x_{a}+\frac{\pi \dot{\alpha}_{m} c}{2 V_{\infty}} e_{m}\right) \cos ^{3} \Lambda c d x \\
\Delta C_{m_{\delta}}=\frac{2}{S} \int_{0}^{L}\left(c_{m_{\delta}}-\frac{x_{a}}{c} c_{L_{\delta}}\right) \delta \cos ^{3} \Lambda c^{2} d x
\end{gathered}
$$

\section{Active Wing Shaping Control}

Since the aeroelasticity can adversely affect the trim drag coefficient at cruise, in order to maintain the best cruise efficiency, the wing shape would need to be actively controlled. A concept of operation is now defined as follows: ${ }^{1}$

- The design point for wing shaping control actuator requirements is defined to be at the half way point of cruise at $30,000 \mathrm{ft}$ corresponding to an aircraft weight of $175,000 \mathrm{lbs}$ with $50 \%$ fuel in the tank.

- At the start of cruise, the aircraft weight is $190,000 \mathrm{lbs}$ with $80 \%$ fuel in the tank. This would correspond to a higher wing loading that causes the wing shape to move away from the optimal wing shape. At the end of cruise, the aircraft weight is $160,000 \mathrm{lbs}$ with $20 \%$ fuel in the tank.

- The VCCTE flap is deployed continuously during cruise to vary the wing shape in order to make the wing more aerodynamically efficient with the highest $L / D$ ratio possible.

The VCCTE flap is designed to shape a wing structure by bending and twist to achieve improved aerodynamic efficiency. The control is accomplished by a slow actuation device since steady state aerodynamics must be established during cruise. Thus, the desired VCCTE flap deflection for wing shaping control can be computed by a trim analysis. The steady-state aeroelastic equations are given by

$$
\begin{aligned}
& \begin{aligned}
\frac{\partial^{2}}{\partial x^{2}}\left(-E B_{2} \gamma^{\prime} \Theta_{x}+E I_{y y} W_{x x}\right) & =-m g+c_{L} q_{\infty} \cos ^{2} \Lambda c \\
+ & \delta\left(x-x_{e}\right)\left[T \sin \Lambda \Gamma-m_{e} g+\left(T \sin \Lambda+m_{e} g \Gamma\right) W_{x}+T \cos \Lambda(\Theta+\gamma)\right] \\
& +\delta\left(x-x_{e}\right)\left(T x_{e} \cos \Lambda+T y_{e} \sin \Lambda+m_{e} g y_{e} \Gamma\right)\left(\Theta_{x}+\gamma^{\prime}\right) \\
& +\delta\left(x-x_{e}\right)\left(-T y_{e} \sin \Lambda \Gamma-T z_{e} \cos \Lambda+m_{e} g y_{e}\right)\left[W_{x x}(\Theta+\gamma)+W_{x}\left(\Theta_{x}+\gamma^{\prime}\right)\right]
\end{aligned} \\
& \begin{aligned}
\frac{\partial}{\partial x}\left\{\left[G J+E B_{1}\left(\gamma^{\prime}\right)^{2}\right] \Theta_{x}-\right. & \left.E B_{2} \gamma^{\prime} W_{x x}\right\}=-m g e_{c g}+c_{m} q_{\infty} \cos ^{2} \Lambda c^{2} \\
- & \delta\left(x-x_{e}\right)\left[-T y_{e} \sin \Lambda \Gamma-T z_{e} \cos \Lambda+m_{e} g y_{e}-\left(T x_{e} \cos \Lambda+T y_{e} \sin \Lambda+m_{e} g y_{e} \Gamma\right) W_{x}\right]
\end{aligned}
\end{aligned}
$$

The steady-state lift and pitching moment coefficients are computed as

$$
\begin{gathered}
c_{L}(x)=c_{L_{\alpha}}(x) \alpha_{a c}(x)+c_{L_{\delta}}(x) \delta(x) \\
c_{m}(x)=c_{m_{a c}}(x)+\frac{e(x)}{c(x)}\left[c_{L_{\alpha}}(x) \alpha_{a c}(x)+c_{L_{\delta}}(x) \delta(x)\right]+c_{m_{\delta}}(x) \delta(x)
\end{gathered}
$$


where the steady-state angle of attack at the aerodynamic center is given by

$$
\alpha_{a c}(x)=\frac{\alpha-\alpha_{i}}{\cos \Lambda}\left(1-\frac{\alpha \gamma}{\cos \Lambda}\right)-\gamma-W_{x} \tan \Lambda\left[1-\frac{\left(\alpha-\alpha_{i}\right) \gamma}{\cos \Lambda}\right]-\Theta\left(1-\frac{\alpha^{2}}{\cos ^{2} \Lambda}\right)+\Theta W_{x} \frac{\left(\alpha-\alpha_{i}\right) \tan \Lambda}{\cos \Lambda}
$$

by setting $\Gamma=0$.

The induced drag of a finite aspect-ratio wing is a consequence of the lift circulation over the wing. For a straight unswept wing, the ideal elliptical lift circulation distribution results in a minimum induced drag whereby the downwash behind the wing is uniform. Suppose a desired optimal lift distribution is known, then let $c_{L}^{*}$ be the optimal lift distribution, $\alpha^{*}$ be the optimal trim angle of attack, and $\alpha_{i}^{*}$ be the optimal induced angle of attack. Using an inverse design method, one can compute the VCCTE flap deflection as a nonlinear feedback control in terms of $\alpha^{*}, W_{x}$, $\Theta$, and $\Theta W_{x}$ according to

$$
\delta\left(\alpha^{*}, W_{x}, \Theta W_{x}\right)=\frac{c_{L}^{*}(x)-c_{L_{\alpha}}(x) \alpha_{a c}^{*}(x)}{c_{L_{\delta}}(x)}
$$

Since $c_{L}^{*}$ is specified, then $c_{m}^{*}$ can be computed as

$$
c_{m}^{*}(x)=c_{m_{a c}}(x)+\frac{e(x)}{c(x)} c_{L}^{*}(x)+c_{m_{\delta}}(x) \frac{c_{L}^{*}(x)-c_{L_{\alpha}}(x) \alpha_{a c}^{*}(x)}{c_{L_{\delta}}(x)}
$$

Then, the steady-state aeroelastic equations can be solved for $W_{x}$ and $\Theta$ explicitly as a function of $x$. Substituting $W_{x}$ and $\Theta$ into the expression for the steady-state angle of attack at the aerodynamic center, the VCCTE flap deflection can be computed explicitly as a function of $x$.

In practice, determining the optimal lift distribution at any point inside a flight envelope can be challenging. Therefore, this problem can be solved by a coupled aerodynamic-aeroelastic optimization whereby the VCCTE flap deflection is optimized to achieve a desired wing shape corresponding to a minimum induced drag.

For example, consider Figure 15 illustrating drag polars of a flexible wing with the VCCTE flap deflection. ${ }^{1}$ The design wing shape corresponds to the one at $50 \%$ fuel remaining at the mid-point of cruise. At the beginning of cruise at $80 \%$ fuel remaining and end of cruise at $20 \%$ fuel remaining, the trim lift coefficients are no longer maintained at the optimal trim lift coefficients. The wing shapes therefore are deflected away from the optimal wing shape. Without wing shaping control, the trim drag at cruise at off-design points can be significant. When the VCCTE flap is applied to bring the wing shape back to the optimal wing shape, for the cruise flight condition at $80 \%$ fuel remaining, the VCCTE flap would deflect upward. The trim drag at this flight condition is slightly greater than that without wing shaping control. Therefore, the control strategy would be to not actuate the VCCTE flap. On the other hand, for the cruise flight condition at $20 \%$ fuel remaining, the VCCTE flap would deflect downward. The trim drag with the VCCTE flap deflection is significantly less than that without wing shaping control. Thus, the VCCTE flap is actuated to reduce the trim drag.

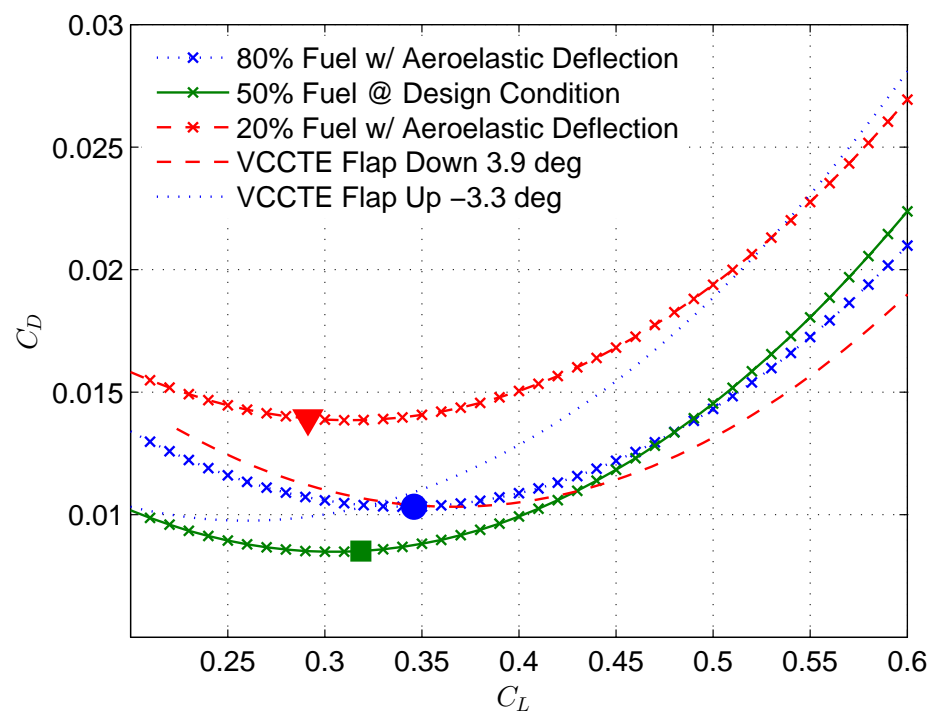

Figure 15 - Drag Polars with VCCTE Flap 
In summary, for aircraft with highly flexible wings, aerodynamic performance can be adversely affected by aeroelasticity. Active wing shaping control is an aerodynamic compensation technique that could result in improved aerodynamic efficiency.

\section{Flutter Analysis}

The aeroelastic equations are expressed in a strong form; i.e., partial differential equations. Weak-form solutions of the equations are expressed as integral forms which can be constructed using various numerical techniques such as finite-element method, Galerkin method, and others. The Galerkin or assumed mode method forms a solution as a superposition of a finite number of eigenfunctions of a simple beam. The finite-element method discretizes the continuous domain in $x$ into discrete elements. A shape or interpolation function is imposed on each element.

A computer model has been developed using the finite-element method. Currently the computer model can compute the frequency and damping as well as static deflections of a wing structure. The model also can compute the aircraft aeroelastic response coupled with rigid-body modes.

The structural rigidities $E I$ and $G J$ are estimated for conventional stiff wing structures of the GTM. For the ESAC, the wing structural rigidities $E I$ and $G J$ are purposely reduced to model highly flexible wing structures. The increased flexibility enables the wing shaping control actuation by the VCCTE flap system.

In addition to the wing dry mass, the fuel mass is also accounted for. The fuel is stored in the center tank and wing main tanks. The center tank holds 20,000 lbs of fuel. Each of the main tanks holds about 15,000 lbs of fuel. The center tank is used first until it is empty. Then, the fuel is drawn equally from the wing main tanks. The fuel mass is modeled as the combined wing mass density. As the structural rigidities are reduced, the wing dry mass also decreases. Assuming that the wing box structure is modeled as a thin-walled structure, then the mass change is related to the change in the wing structural rigidity $E I$ according to Figure 16.

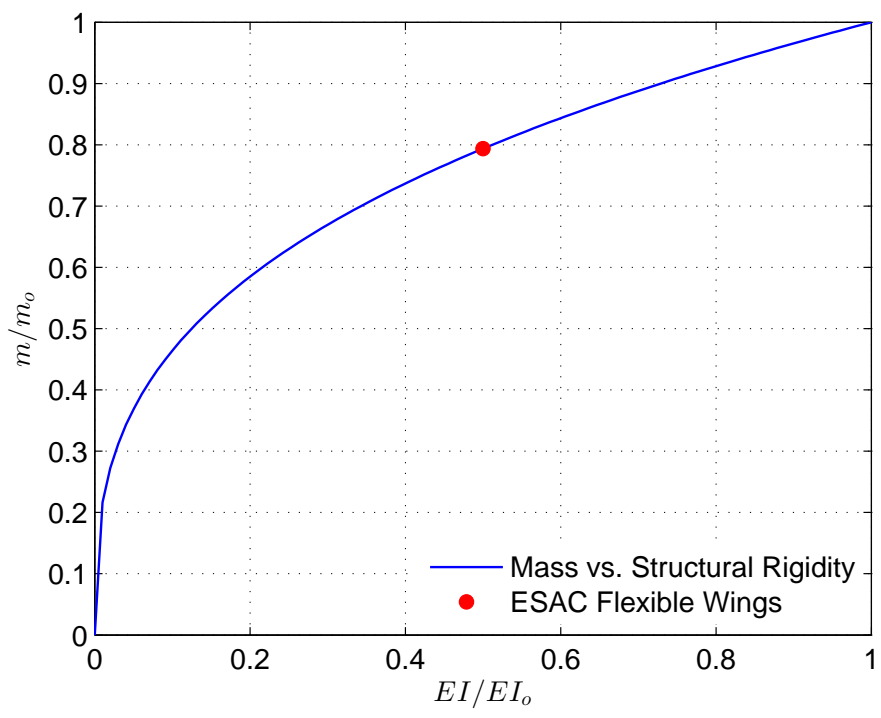

Figure 16 - Mass vs. Structural Rigidity

The structural dynamic modes of the GTM with stiff wings and the ESAC with flexible wings at $80 \%$ fuel remaining are presented in Tables 1 and 2. The symmetric and anti-symmetric mode shapes are plotted in Figures 17 and 18. The frequencies of the ESAC are reduced by $24 \%$ relative to those of the GTM due to the reduced structural rigidities.

The flutter speed prediction is computed using a linear aeroelastic model with an assumed $1 \%$ damping. The frequencies and damping ratios of the first four symmetric and anti-symmetric modes for the GTM with stiff wings and the ESAC with highly flexible wings are plotted in Figures 19 to 22 as a function of the equivalent airspeed in knots. The critical flutter mode is the second bending anti-symmetric mode. The predicted flutter speeds are shown in Tables 3 and 4. Figure 23 shows the flutter boundaries on the flight envelope for the GTM and ESAC. For the GTM, the flutter boundary are well above the maximum airspeed at high altitude, but the flutter boundary for the ESAC with highly flexible wings are within the flight envelope at high altitude. The reduction in the structural rigidities causes 
a significant $29 \%$ drop in the flutter speed. To enable this concept, active flutter suppression control would likely be required.

\begin{tabular}{|c|c|c|c|c|c|}
\hline Mode & Cantilever Mode & Mode & Symmetric Mode & Mode & Anti-Symmetric Mode \\
\hline \hline 1B & 1.3596 & 1B & 1.4338 & 1B & 2.3467 \\
\hline 2B & 3.7405 & 2B & 4.1458 & 2B & 4.3097 \\
\hline 3B & 4.6170 & 3B & 8.2983 & 3B & 6.5671 \\
\hline 1T & 8.7240 & 1T & 9.2571 & 1T & 9.5964 \\
\hline 2T & 9.7822 & 2T & 10.5342 & 4B & 13.1691 \\
\hline 3T & 15.5413 & 3T & 15.3889 & 2T & 15.7262 \\
\hline 4B & 16.9714 & 4B & 16.0972 & 5B & 21.9920 \\
\hline 4T & 23.8500 & 4T & 23.8652 & 3T & 23.7430 \\
\hline 5B & 26.2621 & 5B & 24.5403 & 6B & 25.9084 \\
\hline 5T & 32.7294 & 5T & 32.3880 & 4T & 31.7611 \\
\hline
\end{tabular}

Table 1 - Frequencies of Normal Modes of GTM with Stiff Wings at 80\% Fuel Remaining

\begin{tabular}{|c|c|c|c|c|c|}
\hline Mode & Cantilever Mode & Mode & Symmetric Mode & Mode & Anti-Symmetric Mode \\
\hline \hline 1B & 1.0340 & 1B & 1.0877 & 1B & 1.7764 \\
\hline 2B & 2.7446 & 2B & 3.1505 & 2B & 3.1650 \\
\hline 3B & 3.4350 & 3B & 6.2524 & 3B & 4.9710 \\
\hline 1T & 6.6018 & 1T & 7.1543 & 1T & 7.4328 \\
\hline 2T & 7.5237 & 2T & 8.0580 & 4B & 9.9622 \\
\hline 3T & 12.0462 & 3T & 11.7414 & 2T & 12.2519 \\
\hline 4B & 12.8829 & 4B & 12.4047 & 5B & 16.8019 \\
\hline 4T & 18.4476 & 4T & 18.4267 & 3T & 18.3986 \\
\hline 5B & 20.0045 & 5B & 18.7751 & 6B & 20.1822 \\
\hline 5T & 25.4651 & 5T & 25.0106 & 4T & 24.1093 \\
\hline
\end{tabular}

Table 2 - Frequencies of Normal Modes of ESAC with Highly Flexible Wings at 80\% Fuel Remaining

\begin{tabular}{|c|c|c|c|}
\hline Altitude, $10^{3} \mathrm{ft}$ & Cantilever Mode & Symmetric Mode & Anti-Symmetric Mode \\
\hline \hline 0 & 0.6499 & 0.6336 & 0.5790 \\
\hline 5 & 0.7103 & 0.6925 & 0.6319 \\
\hline 10 & 0.7792 & 0.7596 & 0.6923 \\
\hline 15 & 0.8583 & 0.8364 & 0.7615 \\
\hline 20 & 0.9496 & 0.9251 & 0.8411 \\
\hline 25 & 1.0555 & 1.0280 & 0.9334 \\
\hline 30 & 1.1795 & 1.1483 & 1.0411 \\
\hline 35 & 1.3253 & 1.2897 & 1.1674 \\
\hline
\end{tabular}

Table 3 - Flutter Speed Prediction for Aeroelastic GTM with Stiff Wings at 80\% Fuel Remaining 


\begin{tabular}{|c|c|c|c|}
\hline Altitude, $10^{3} \mathrm{ft}$ & Cantilever Mode & Symmetric Mode & Anti-Symmetric Mode \\
\hline \hline 0 & 0.4612 & 0.4554 & 0.4156 \\
\hline 5 & 0.5037 & 0.4974 & 0.4530 \\
\hline 10 & 0.5522 & 0.5451 & 0.4956 \\
\hline 15 & 0.6078 & 0.6000 & 0.5445 \\
\hline 20 & 0.6718 & 0.6629 & 0.6008 \\
\hline 25 & 0.7461 & 0.7360 & 0.6658 \\
\hline 30 & 0.8329 & 0.8214 & 0.7418 \\
\hline 35 & 0.9350 & 0.9218 & 0.8308 \\
\hline
\end{tabular}

Table 4 - Flutter Speed Prediction for ESAC with Highly Flexible Wings at 80\% Fuel Remaining

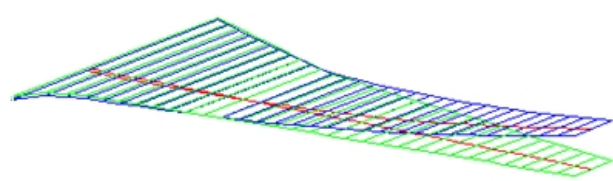

Mode 1B

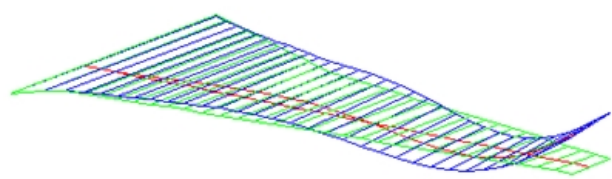

Mode 3B

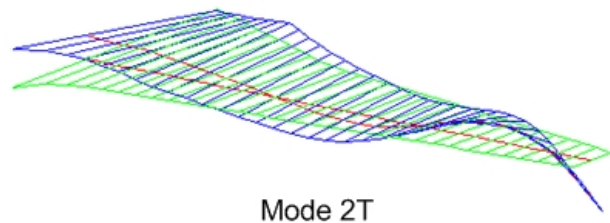

Mode 2T

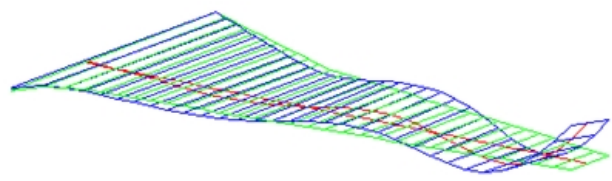

Mode 4B

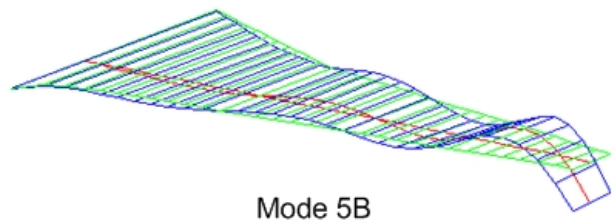

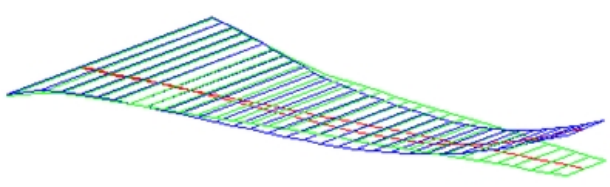

Mode 2B

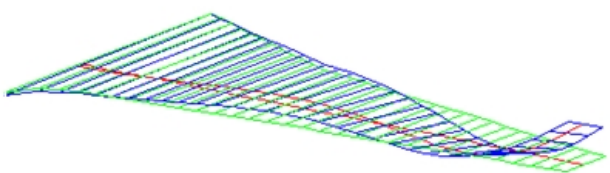

Mode 1T

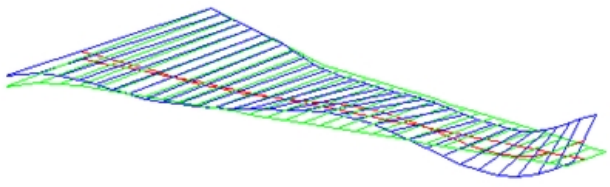

Mode 3T

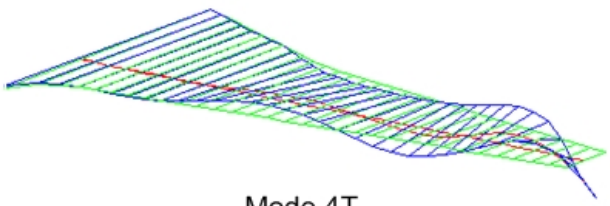

Mode 4T

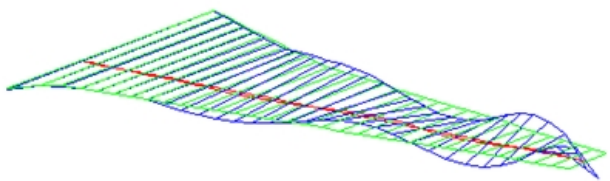

Mode 4T

Figure 17- Symmetric Mode Shapes 


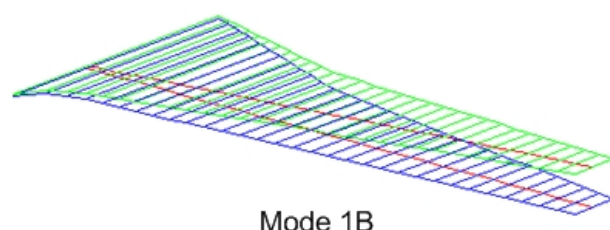

Mode 1B

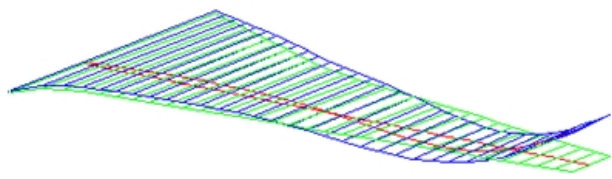

Mode 3B

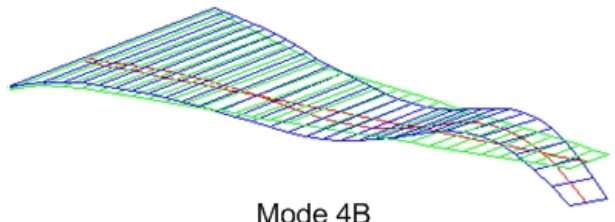

Mode 4B

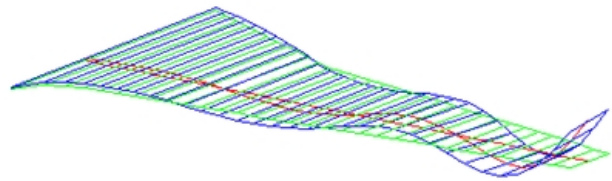

Mode 5B

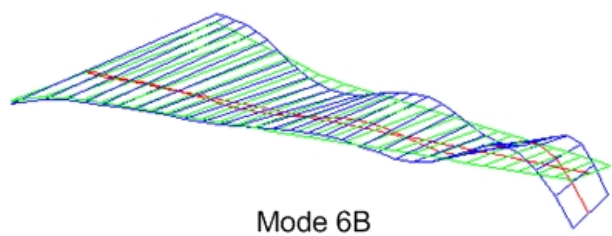

Figure 18 - Anti-Symmetric Mode Shapes

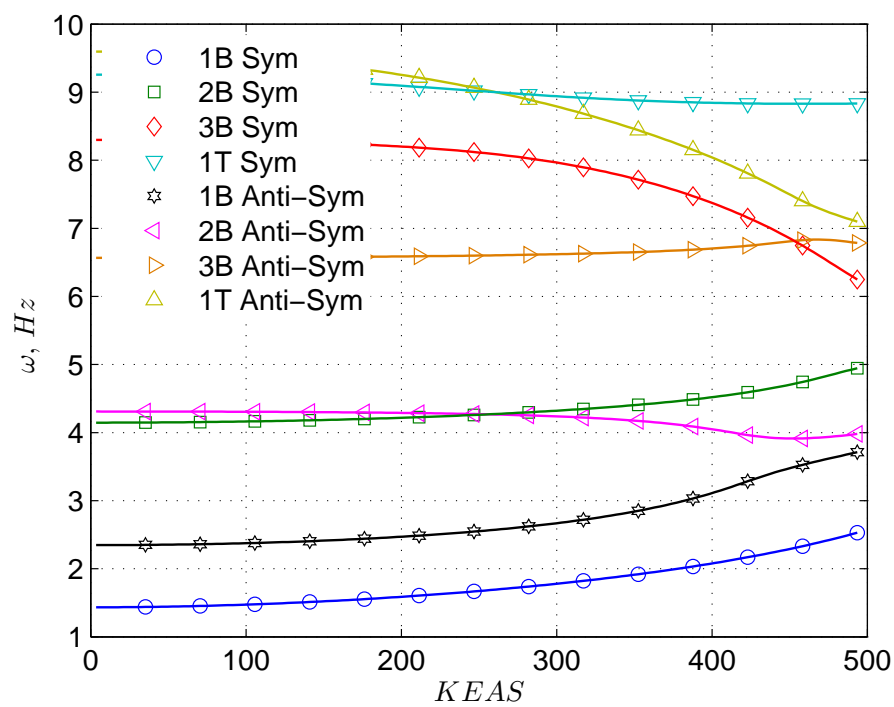

Figure 19 - Frequencies of GTM with Stiff Wings at 35,000 ft
Mode 2B
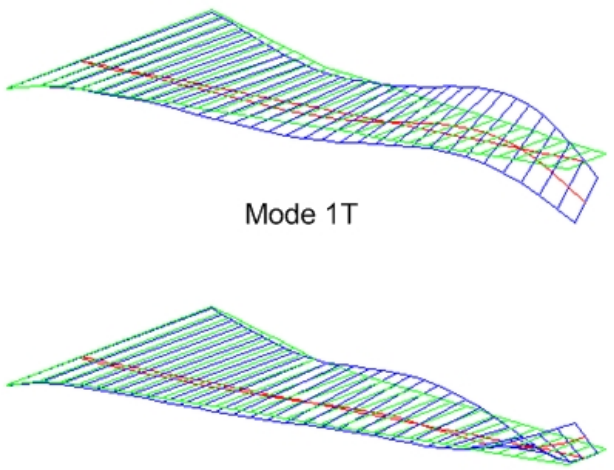

Mode 2T
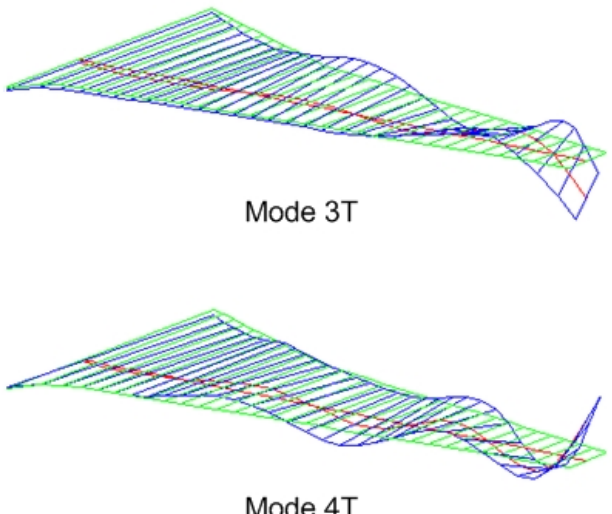

Mode 4T 


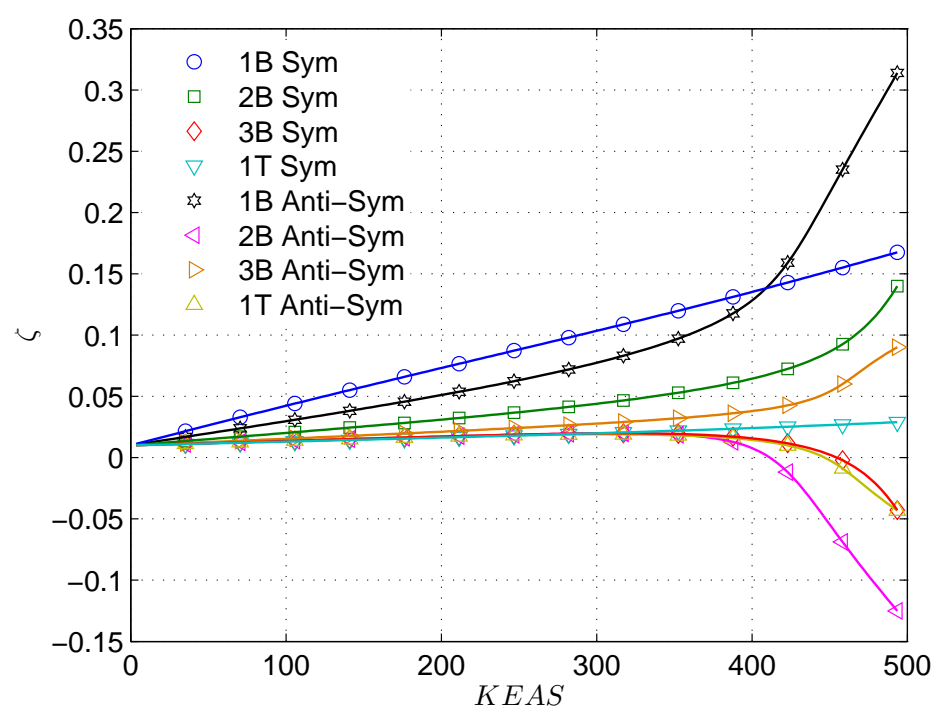

Figure 20 - Damping Ratios of GTM with Stiff Wings at 35,000 ft

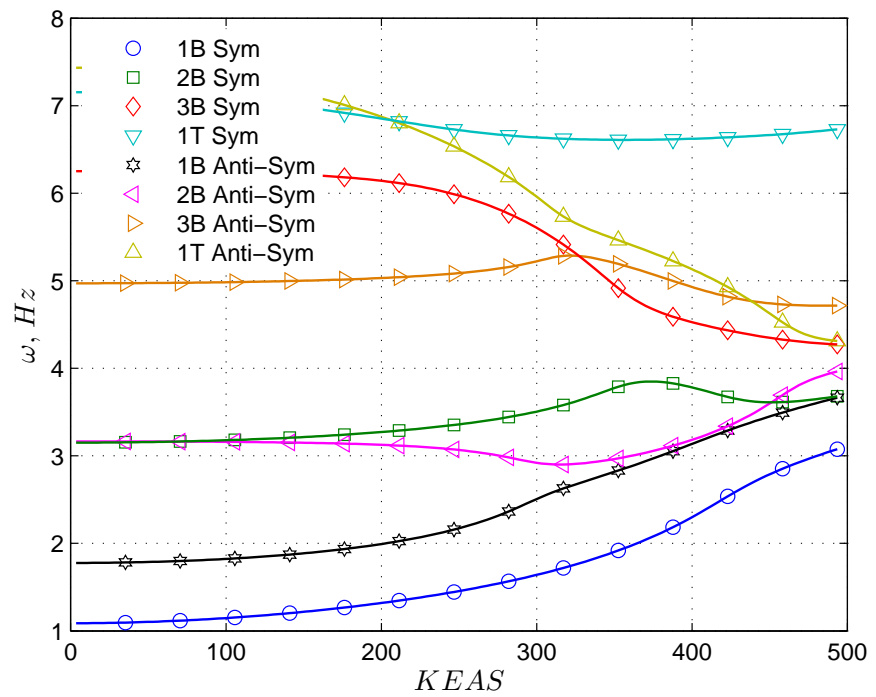

Figure 21 - Frequencies of ESAC with Highly Flexible Wings at 35,000 ft 


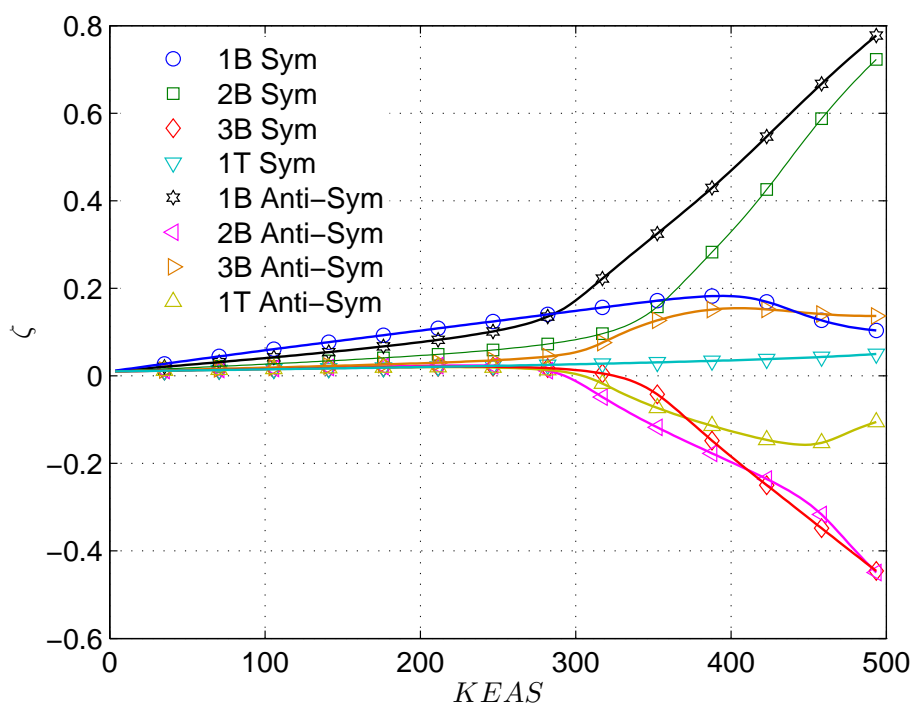

Figure 22 - Damping Ratios of ESAC with Highly Flexible Wings at 35,000 ft

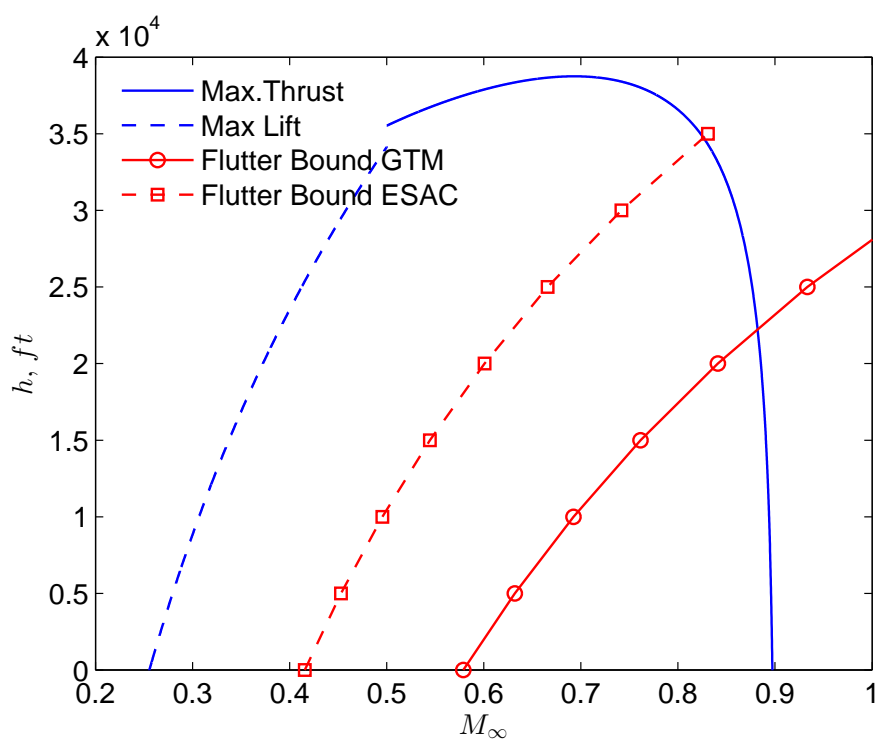

Figure 23 - Flight Envelope and Flutter Boundaries of GTM and ESAC

\section{Multi-Objective Flight Control for Drag Reduction}

Flight control design is an enabling feature of the ESAC. A typical flight control design usually takes into account different sets of requirements for performance and stability that must be considered during a design process. Performance in the context of flight control implies the ability for a flight control system to follow a pilot command. However, in this study, a new notion of aerodynamic performance is introduced into the flight control framework. The goal of the new vehicle is to achieve low drag through active wing shaping control actuation. Thus, drag penalty due to the VCCTE flap deflection should be considered in a flight control design. Hence, a new concept of multi-objective flight control is proposed to not only achieve a pilot command following objective but also a drag reduction objective ${ }^{1}$ during maneuvers such as a pitch command or roll command.

Stability is of paramount importance for any flight vehicle. Structural flexibility of airframes including wings can cause significant aeroelastic interactions that can degrade vehicle stability margins, potentially leading to loss of control. There exists a trade-off between the desire of having light weight, flexible structures for weight savings 
and the need for maintaining sufficient robust stability margins from aeroelastic instability. In this study, as can be seen, the flutter speed occurs at a relatively low Mach number. Thus, a flight control system must be able to stabilize aeroelastic modes. The VCCTE flap system must be designed to achieve this objective. Clearly, it is generally not desirable to operate an unstable vehicle. Thus, in practice, aeroelastic tailoring in the design process can be used to increase aerodynamic and structural damping. Then, the role of a flight control system would be relegated to stability augmentation as opposed to a more demanding task of stabilization. This is acceptable in the current practices as there are many stability augmentation controllers such as yaw dampers in use to provide desired damping characteristics to meet pilot handling quality requirements.

Gust load alleviation control is also an important part of the overall flight control strategy for flexible aircraft. As flexibility increases, the vehicle aeroelastic response to wind gust disturbances can result in handling quality issues as well as ride quality problems. Gust load alleviation control will reduce the aeroelastic response by reactive feedback control or predictive feedforward control using early detection turbulence sensors. For reactive feedback control, adaptive control can potentially provide an effective solution. In a previous work, adaptive control for gust load alleviation has been investigated using two recently developed adaptive control methods. ${ }^{11}$

The variable camber feature of the VCCTE flap is created by the three chordwise flap segments. For flight control functions, the outermost chordwise flap segment is designed to be a fast acting control surface. This flap segment is spanned the entire wing and is assumed to have sufficient bandwidth and control power for roll control and flutter suppression control as well as drag reduction control.

Because the VCCTE flap is a piecewise continuous control surface, so it needs to be treated in a special way. Suppose the ideal continuous trailing edge of the VCCTE flap is mathematically smooth and can be described by a Fourier sine series as follows:

$$
\delta^{*}(x)=A_{0}+\sum_{n=1}^{N} A_{n} \sin \frac{n \pi x}{L}
$$

where $\delta^{*}(x)$ is a theoretical VCCTE flap deflection and $N$ is the number of terms in the Fourier sine series

In practice, the trailing edge of the VCCTE flap is only piecewise continuous due to a physical implementation. Therefore, the actual VCCTE flap deflection is a piecewise linear approximation of the theoretical VCCTE flap deflection. Thus

$$
\delta(x)=A_{0}+\frac{x}{x_{i+1}-x_{i}} \sum_{n=1}^{N} A_{n}\left(\sin \frac{n \pi x_{i+1}}{L}-\sin \frac{n \pi x_{i}}{L}\right), x_{i} \leq x<x_{i+1}
$$

where $x_{i}, i=1,2, . m$, is the location of one of the two edges of a spanwise flap segment, and $m$ is the number of spanwise segments of the VCCTE flap deflection.

Note that $A_{0}$ is the constant flap deflection that is needed to provide flexibility for multi-objective flight control, especially for drag reduction control. The constant flap deflection at the wing tip can be used to create a zero lift condition to minimize tip vortices. The quantities $A_{n}, n=0,1, \ldots, N$ can be viewed as virtual control variables. The virtual control variables are then used in a flight control design to determine their command settings. Then, the actual VCCTE flap deflection command $\delta\left(x_{i}\right)$ can be computed from the virtual control commands $A_{n}$.

It is assumed that the distributed aeroelastic system is converted into a finite-dimensional system using the Galerkin method or finite-element method. Then the state space representation of the aeroservoelastic flight dynamic model can be expressed as

$$
\left[\begin{array}{c}
\dot{x}_{e} \\
\dot{x}_{r}
\end{array}\right]=\left[\begin{array}{cc}
A_{e} & A_{e r} \\
A_{r e} & A_{r}
\end{array}\right]\left[\begin{array}{c}
x_{e} \\
x_{r}
\end{array}\right]+\left[\begin{array}{cc}
B_{e} & B_{e r} \\
B_{r e} & B_{r}
\end{array}\right]\left[\begin{array}{c}
u_{e} \\
u_{r}
\end{array}\right]
$$

where $x_{e}$ is the wing aeroelastic state vector, $x_{a}$ is the rigid-body aircraft state vector, $u_{e}=\left[\begin{array}{llll}A_{0} & A_{1} & \ldots & A_{N}\end{array}\right]^{\top}$ is the virtual control vector, and $u_{r}$ is the elevator deflection.

Generally, the aeroelastic state vectors are not accessible but can be estimated by an observer design which is a standard control practice. It is assumed that sufficient aeroelastic deflection sensors such as accelerometers or fiber optics sensors are available for the observer design. Alternatively, it is possible to reconstruct the aeroelastic deflections from limited sensor measurements by a model-based approach. Assuming that the aeroelastic states can be reconstructed by an observer design for feedback control and that the estimation error is sufficiently small, then a flight control task can be designed with the estimated aeroelastic states as feedback state variables.

As an example, consider a flight control design for an angle of attack command. Let $x_{a}=\int_{0}^{t} \Delta \alpha d \tau$ be an integral error state of the angle of attack, where $\Delta \alpha=\alpha-\alpha_{c}$ is the error between the angle of attack and its command signal. The augmented plant is expressed as 


$$
\dot{x}_{a}=A_{a}\left(x_{r}-x_{c}\right)
$$

where $x_{c}$ is a command vector.

$$
\begin{gathered}
\text { Let } x=\left[\begin{array}{ccc}
x_{e} & x_{r} & x_{a}
\end{array}\right]^{\top} \text { and } u=\left[\begin{array}{ll}
u_{e} & u_{r}
\end{array}\right]^{\top} . \text { Then the augmented plant is expressed as } \\
\dot{x}=A x+B u+z
\end{gathered}
$$

where

$$
A=\left[\begin{array}{ccc}
A_{e} & A_{e r} & 0 \\
A_{r e} & A_{r} & 0 \\
0 & A_{a} & 0
\end{array}\right], B=\left[\begin{array}{cc}
B_{e} & B_{e r} \\
B_{r e} & B_{r} \\
0 & 0
\end{array}\right], z=\left[\begin{array}{c}
0 \\
0 \\
-A_{a} x_{c}
\end{array}\right]
$$

An optimal control is designed with the following multi-objective cost function

$$
J=\frac{1}{2} \int_{0}^{t_{f}}\left(x_{a}^{\top} Q_{a} x_{a}+x_{e}^{\top} Q_{e} x+u^{\top} R u+q_{D} C_{D}\right) d t
$$

The first term in the cost function is designed to track the command signal. The second term in the cost function is designed to stabilize any aeroelastic modes. The third term is designed to minimize the control effort. Hence, the first three terms in the cost function form the standard linear-quadratic regulator (LQR) cost function. The last term is designed to minimize the drag coefficient with $q_{D}$ a weighting constant. It is assumed that the drag coefficient can be estimated accurately via a drag polar model for which the drag polar parameters are known. The drag polar model can be expressed as

$$
C_{D}=C_{D_{0}}+K\left(C_{L_{0}}+C_{L_{x}} x+C_{L_{u}} u\right)^{2}+C_{D_{u}} u+u^{\top} C_{D_{u^{2}}} u
$$

where $K$ is the drag polar parameter, $C_{L_{x}}$ is the lift stability derivative row vector, $C_{L_{u}}$ is the lift control derivative row vector, $C_{D_{u}}$ is the drag control derivative row vector, and $C_{D_{u^{2}}}$ is the positive-definite quadratic drag control derivative matrix.

The cost function can now be expressed as

$$
J=\frac{1}{2} \int_{0}^{t_{f}}\left[x^{\top} Q x+u^{\top} R u+q_{D} C_{D_{0}}+q_{D} K\left(C_{L_{0}}+C_{L_{x}} x+C_{L_{u}} u\right)^{2}+q_{D} C_{D_{u}} u+u^{\top} q_{D} C_{D_{u^{2}}} u\right] d t
$$

where $Q=\operatorname{diag}\left(Q_{e}, 0, Q_{a}\right)$.

The Hamiltonian function of the optimal control problem is defined as

$$
H=\frac{1}{2}\left[x^{\top} Q x+u^{\top} R u+q_{D} C_{D_{0}}+q_{D} K\left(C_{L_{0}}+C_{L_{x}} x+C_{L_{u}} u\right)^{2}+q_{D} C_{D_{u}} u+u^{\top} q_{D} C_{D_{u^{2}}} u\right]+\lambda^{\top}(A x+B u+z)
$$

where $\lambda$ is the adjoint vector.

The adjoint equation is obtained as

$$
\dot{\lambda}=-\frac{\partial H^{\top}}{\partial x}=-Q x-q_{D} K C_{L_{x}}^{\top}\left(C_{L_{0}}+C_{L_{x}} x+C_{L_{u}} u\right)-A^{\top} \lambda
$$

The optimality condition is

$$
\frac{\partial H^{\top}}{\partial u}=0=R u+q_{D} K C_{L_{u}}^{\top}\left(C_{L_{0}}+C_{L_{x}} x+C_{L_{u}} u\right)+\frac{1}{2} q_{D} C_{D_{u}}^{\top}+q_{D} C_{D_{u^{2}}} u+B^{\top} \lambda
$$

Solving for the optimal control yields

$$
u=-\left(R+q_{D} K C_{L_{u}}^{\top} C_{L_{u}}+q_{D} C_{D_{u^{2}}}\right)^{-1}\left(q_{D} K C_{L_{u}}^{\top} C_{L_{0}}+\frac{1}{2} q_{D} C_{D_{u}}^{\top}+q_{D} K C_{L_{u}}^{\top} C_{L_{x}} x+B^{\top} \lambda\right)
$$

To solve for these equations, the adjoint equation and the state equation must be solved simultaneously along with the optimal control. Let $\lambda=P x+S z+\lambda_{0}$ be a solution of the adjoint vector. Then, the adjoint equation is obtained as

$$
\begin{aligned}
& \dot{P} x+P \dot{x}+\dot{S} z+S \dot{z}=-\left(Q+q_{D} K C_{L_{x}}^{\top} C_{L_{x}}+A^{\top} P\right) x-A^{\top} S z-q_{D} K q_{D} K C_{L_{x}}^{\top} C_{L_{0}}-A^{\top} \lambda_{0} \\
& +q_{D} K C_{L_{x}}^{\top} C_{L_{u}}\left(R+q_{D} K C_{L_{u}}^{\top} C_{L_{u}}+q_{D} C_{D_{u^{2}}}\right)^{-1}\left(q_{D} K C_{L_{u}}^{\top} C_{L_{0}}+\frac{1}{2} q_{D} C_{D_{u}}^{\top}+q_{D} K C_{L_{u}}^{\top} C_{L_{x}} x+B^{\top} P x+B^{\top} S z+B^{\top} \lambda_{0}\right)
\end{aligned}
$$


Let $\alpha_{c}$ be a step input so that $\dot{\alpha}_{c}=0$. Let $t_{f} \rightarrow \infty$, then the optimal solution approaches a steady state solution. Therefore, $\dot{P}(0)=0$ and $\dot{S}(0)=0$. Then, separating terms yields the following expressions

$$
\begin{gathered}
P A+A^{\top} P-\left(P B+q_{D} K C_{L_{x}}^{\top} C_{L_{u}}\right)\left(R+q_{D} K C_{L_{u}}^{\top} C_{L_{u}}+q_{D} C_{D_{u^{2}}}\right)^{-1}\left(B^{\top} P+q_{D} K C_{L_{u}}^{\top} C_{L_{x}}\right)+Q+q_{D} K C_{L_{x}}^{\top} C_{L_{x}}=0 \\
S=\left[\left(P B+q_{D} K C_{L_{x}}^{\top} C_{L_{u}}\right)\left(R+q_{D} K C_{L_{u}}^{\top} C_{L_{u}}+q_{D} C_{D_{u^{2}}}\right)^{-1} B^{\top}-A^{\top}\right]^{-1} P \\
\lambda_{0}=\left[\left(P B+q_{D} K C_{L_{x}}^{\top} C_{L_{u}}\right)\left(R+q_{D} K C_{L_{u}}^{\top} C_{L_{u}}+q_{D} C_{D_{u^{2}}}\right)^{-1} B^{\top}-A^{\top}\right]^{-1} \times \\
\times\left[q_{D} K C_{L_{x}}^{\top} C_{L_{0}}-\left(P B+q_{D} K C_{L_{x}}^{\top} C_{L_{u}}\right)\left(R+q_{D} K C_{L_{u}}^{\top} C_{L_{u}}+q_{D} C_{D_{u^{2}}}\right)^{-1}\left(q_{D} K C_{L_{u}}^{\top} C_{L_{0}}+\frac{1}{2} q_{D} C_{D_{u}}^{\top}\right)\right]
\end{gathered}
$$

Equation (126) is a Ricatti equation expressed in a non-standard form. It can be recast in a standard form as

$$
P \bar{A}+\bar{A}^{\top} P-P B \bar{R}^{-1} B^{\top} P+\bar{Q}=0
$$

where

$$
\begin{gathered}
\bar{A}=A-B \bar{R}^{-1} q_{D} K C_{L_{u}}^{\top} C_{L_{x}} \\
\bar{R}=R+q_{D} K C_{L_{u}}^{\top} C_{L_{u}}+q_{D} C_{D_{u^{2}}} \\
\bar{Q}=Q+q_{D} K C_{L_{x}}^{\top} C_{L_{x}}\left(1-\bar{R}^{-1} q_{D} K C_{L_{u}}^{\top} C_{L_{x}}\right)
\end{gathered}
$$

The optimal control is then given by

$$
u=\bar{R}^{-1}\left[q_{D} K C_{L_{u}}^{\top} C_{L_{0}}+\frac{1}{2} q_{D} C_{D_{u}}^{\top}+B^{\top} \lambda_{0}+\left(B^{\top} P+q_{D} K C_{L_{u}}^{\top} C_{L_{x}}\right) x+B^{\top} S z\right]
$$

Thus, an optimal control framework has been developed to address the drag reduction control during maneuvers for an aircraft with highly flexible wings. The multi-objective flight control addresses simultaneously pilot command tracking tasks, aeroelastic stabilization, and drag reduction control simultaneously. In future studies, we will apply this approach to demonstrate the multi-objective flight control for drag reduction.

\section{Discussions and Recommendations}

The 2010 NASA Innovation Fund Award study has identified some potentially promising concepts of aerodynamically efficient elastic wing shapes, wing shaping control, and the VCCTE flap. The potential drag reduction for these concepts could be significant. In terms of new revolutionary concepts such as non-planar elastic wing shapes and the VCCTE flap, current constraints in aircraft development capabilities may preclude such concepts to be adopted due to potential technical challenges in many different areas. One obvious area is materials and structure. To achieve the continuous trailing edge, radical changes in materials and structures would have to occur. Advances in composites material development may be able to address some of the challenges in materials and structures.

The study shows that to harvest the potential of wing shaping control, configuration changes in high-lift devices would have to be a part of the wing shaping control strategy. Flap and slat devices inherently generate drag as they increase lift. Conventional flap and slat systems as in the current generation aircraft are not aerodynamically efficient enough for drag-reducing control strategies like wing shaping control. The variable camber continuous trailing flap concept developed in this study does seem to offer a potential pay-off for drag reduction even when used in current generation aircraft. Technical challenges would exist as the increase in the number of multiple segmented flaps that form a variable camber continuous flap surface would lead to increased design complexity.

The issues of wing flexibility on vehicle stability cannot be ignored. No matter how aerodynamically efficient an aircraft would be, it may never fly if it is unstable. Flight control can be used to stabilize aeroelastic instability that may be associated with wing flexibility. Aeroelastic tailoring by properly distributing wing stiffness throughout the airframe may also improve stability margins of aeroelastic modes. The role of flight control is then relegated to stability augmentation which would reduce the demand on a flight control system. In any case, increased wing flexibility would result in more susceptibility to potentially adverse responses to air turbulence and wind gusts. Flight 
control design would need to take this issue into consideration. Gust load alleviation control technology has been deployed on modern commercial aircraft. Such technology may become standard one day for aircraft flight control design as the trend in aircraft design is moving toward a more flexible airframe design for increased performance and reduced fuel burn.

The 2010 NASA Innovation Fund Award study was conducted jointly between NASA and Boeing Research and Technology. Currently, this work is being supported by NASA Subsonic Fixed Wing (SFW) Project to develop these initial concepts further. Boeing Research and Technology is currently collaborating with NASA to develop the VCCTE flap system. Toward this end, Boeing Research and Technology recommendations for use of the variable camber continuous trailing edge flap concept include:

1. Roll Control - During cruise and during take-off and landing, a combination of flaps can be applied to roll the aircraft at required rates. A balance of leading and trailing edge flaps can be used to ensure that the twist of the flexible wing does not result in roll reversal. With use of an adaptive or feedback gain schedule, a very consistent roll rate per stick command can be achieved. Another way to roll a flexible wing aircraft is to use some of the leading edge control surfaces to establish a twist in the wing in the direction of the desired roll. This method was flight-demonstrated in the NASA/Boeing Active Aeroelastic Wing (AAW) program. A NASA F/A18 was configured with a flexible wing, and an outboard leading edge flap used to twist the wing for roll control. The horizontal stabilator was set for symmetric pitch response only. Usually the stabilators are dominant in rolling the aircraft at higher speeds due to the roll reversal using ailerons. The AAW program quite successfully demonstrated effective roll control.

2. Lift Change - Lift can be increased for take-off and landing; the VCCTE flap deploys to effect a wing airfoil shape that can increase the lift for a given angle of attack. After take-off, the high lift configuration can be altered for varying VCCTE flap deployment, thus gradually reducing drag during the climb-out to cruise conditions. For cruise flight, the VCCTE flap and slat can be used to change the wing spanwise lift distribution so that a minimum drag profile can continually be achieved. This may involve both the variable flap position and the twist of the wing as a function of span.

3. Stabilization of Wing Flexible Modes - Flight control modes can be installed that suppress wing structure's oscillations that may be due to the flexible design of the wing. Both wing vertical bending and wing torsional modes can be suppressed. The flap actuation rates and dynamics must have sufficient bandpass to deal with the higher frequency wing flexibility modes. This requires actuation drive motors that can provide sufficient acceleration to achieve the higher bandpass. These leading and trailing control surfaces will act to reduce the oscillation magnitudes. Not all of the control surfaces are needed for this function. Remaining surfaces not used for this task can have a lower controller bandpass. Acceleration sensors mounted at the maximum deflection location on the wing will provide the control commands sent to certain selected leading and trailing edge surfaces that will suppress the structural mode oscillations to acceptable values. This can be done by changing the actuator command phase angle so that the resulting surface response counter balances the phase of the oscillations.

4. Variable Camber Continuous Trailing Edge Flap Design for Optimum Control Drag Reduction - Conventional flaps for each segment on the wing trailing edge may result in increased drag at cruise conditions for large flap deflection. A method to segment the flap into multiple sections results in a variable flap camber that provides the necessary force but with less flap deflection and consequently less drag due to the wing control surfaces. Each flap segment would have sufficient flexibility to exactly fit the end of the adjacent flap resulting in a continuous flap surface for cruise.

\section{Conclusions}

This study introduces a new aircraft concept called Elastically Shaped Aircraft Concept (ESAC). The aircraft concept addresses the drag reduction goal in commercial aviation through an elastic wing shaping control approach for aircraft with highly flexible wing structures. The multi-disciplinary nature of flight physics is appreciated with the recognition of the potential adverse effects of aeroelastic wing shape deflections on aerodynamic drag. By aeroelastically tailoring the wing shape with active control, a significant drag reduction benefit could be realized. To attain the potential of the elastic wing shaping control concept, a new type of aerodynamic control effector is introduced. This effector concept is referred to as a Variable Camber Continuous Trailing Edge (VCCTE) flap. Conventional aerodynamic control surfaces are discrete independent surfaces that cause geometric discontinuities at the trailing edge 
region. These discontinuities promote vortices which result in drag rises. The VCCTE flap concept could provide a substantial drag reduction benefit over a conventional discrete flap system. Aerodynamic simulations show a drag reduction of over 50\% could be achieved with the flap concept over a conventional discrete flap system. Moreover, elastic wing shaping control potential could only be realized with low drag aerodynamic control surfaces such as this flap concept.

To enable the wing shaping control technology, this study develops a nonlinear aeroelastic model for the ESAC. The planform of the ESAC is based on the NASA Generic Transport Model (GTM), but the ESAC wing structure is modeled with a significant reduction in the structural rigidities. A flutter analysis is conducted to compute the frequencies, damping ratios, and mode shapes of the aeroelastic modes of the wing structure. Flutter boundaries for the GTM and ESAC are predicted. Critical flutter modes are associated with the anti-symmetric modes. The flutter boundary of the GTM is sufficiently far way from the flight envelope. On the other hand, the flutter boundary of the ESAC encroaches the flight envelope at high altitude. This implies that flutter suppression control would be needed to improve the flutter margin.

A concept of multi-objective flight control for drag reduction is introduced to address a conventional flight control objective of pilot command tracking tasks in combination with other flight control objectives of aeroelastic stabilization and drag reduction control. An optimal control framework is developed and will be applied in future studies to demonstrate the potential benefit of the multi-objective flight control for drag reduction during maneuvers.

\section{Acknowledgment}

The authors wish to acknowledge Mr. John Dykman and Mr. Brian Foist of Boeing Research \& Technology in Seal Beach, CA for providing technical insights and support of the aeroelastic model development. The authors also would like to thank the NASA Aeronautics Research Mission Directorate (ARMD) Subsonic Fixed Wing Project under the Fundamental Aeronautics Program for providing the funding support of this work.

\section{References}

\footnotetext{
${ }^{1}$ Nguyen, N., "NASA Innovation Fund 2010 Project: Elastically Shaped Future Air Vehicle Concept," NASA Internal Report Submitted to NASA Innovative Partnerships Program Office, October 8, 2010.

${ }^{2}$ Jordan, T. L., Langford, W. M., Belcastro, C. M., Foster, J. M., Shah, G. H., Howland, G., and Kidd, R., "Development of a Dynamically Scaled Generic Transport Model Testbed for Flight Research Experiments," AUVSI Unmanned Unlimited, Arlington, VA, 2004.

${ }^{3}$ Nguyen, N., Nelson, A., Pulliams, T., "Damage Adaptive Control System Research Report,” Internal NASA Report, April 2006.

${ }^{4}$ Stanford University, AA241, "Aircraft Design: Synthesis and Analysis," http://adg.stanford.edu/aa241/structures/weightstatements.html

${ }^{5}$ Warwick, G., "Flying Greener, on the Wings of a Gull", Aviation Week, Leading Edge AW\&ST On Technology, May $26,2011$.

${ }^{6}$ Nguyen, N., "Integrated Flight Dynamics Modeling of Flexible Aircraft with Inertial Force-Propulsion - Aeroelastic Couplings," 46th AIAA Aerospace Sciences Meeting and Exhibit, AIAA-2008-194, January 2008.

${ }^{7}$ Nguyen, N., Trinh, K., Nguyen, D., Tuzcu, I., "Nonlinear Aeroelasticity of Flexible Wing Structure Coupled with Aircraft Flight Dynamics," AIAA Structures, Structural Dynamics, and Materials Conference, AIAA-2012-1792, April 2012.

${ }^{8}$ Etkin, B., Dynamics of Flight - Stability and Control, John Wiley \& Sons, Inc. 1959.

${ }^{9}$ Theodorsen, T., Garrick, I., "Mechanism of Flutter - a Theoretical and Experimental Investigation of the Flutter Problem", NACA Report 685, 1940.

${ }^{10}$ Houbolt, J., Brooks, G., "Differential Equations of Motion for Combined Flapwise Bending, Chordwise Bending, and Torsion of Twisted Nonuniform Rotor Blades," NACA Technical Note 3905, February 1957.

${ }^{11}$ Nguyen, N., Tuzcu, I., Yucelen, T., Calise, A., "Longitudinal Dynamics and Adaptive Control Application for an Aeroelastic Generic Transport Model,” AIAA Atmospheric Flight Mechanics Conference, AIAA-2011-6319, August 2011.
} 